\title{
2002 Guidelines for the Use of Antimicrobial Agents in Neutropenic Patients with Cancer
}

\author{
Walter T. Hughes, ${ }^{1}$ Donald Armstrong, ${ }^{2}$ Gerald P. Bodey, ${ }^{3}$ Eric J. Bow, ${ }^{7}$ Arthur E. Brown, ${ }^{2}$ Thierry Calandra, ${ }^{9}$ \\ Ronald Feld, ${ }^{8}$ Philip A. Pizzo, ${ }^{4,5}$ Kenneth V. I. Rolston, ${ }^{3}$ Jerry L. Shenep, ${ }^{1}$ and Lowell S. Young ${ }^{6}$ \\ ${ }^{1}$ St. Jude Children's Research Hospital, Memphis, Tennessee; ${ }^{2}$ Memorial Sloan-Kettering Cancer Center, New York, New York; ${ }^{3}$ University of Texas \\ M. D. Anderson Cancer Center, Houston; ${ }^{4}$ Harvard Medical School, Boston, Massachusetts; ${ }^{5}$ Stanford University School of Medicine, Palo Alto, \\ and ${ }^{6}$ Kuzell Institute for Arthritis, San Francisco, California; ${ }^{7}$ University of Manitoba, Winnipeg, and ${ }^{8}$ Princess Margaret Hospital, Toronto, Canada; \\ and ${ }^{9}$ Centre Hospitalier Universitaire Vaudois, Lausanne, Switzerland
}

\section{EXECUTIVE SUMMARY}

This article, prepared by the Infectious Diseases Society of America (IDSA) Fever and Neutropenia Guidelines Panel, updates guidelines established a decade ago by the Infectious Disease Society of America for the use of antimicrobial agents to treat neutropenic patients with unexplained fever [1].

\section{Definitions}

Fever is defined as a single oral temperature of $\geqslant 38.3^{\circ} \mathrm{C}$ $\left(101^{\circ} \mathrm{F}\right)$ or a temperature of $\geqslant 38.0^{\circ} \mathrm{C}\left(100.4^{\circ} \mathrm{F}\right)$ for $\geqslant 1$ h. Neutropenia is defined as a neutrophil count of $<500$ cells $/ \mathrm{mm}^{3}$, or a count of $<1000$ cells $/ \mathrm{mm}^{3}$ with a predicted decrease to $<500$ cells $/ \mathrm{mm}^{3}$.

\section{Initial Evaluation}

Determine whether the patient is at low risk for complications; determine whether vancomycin therapy is needed.

\section{Initial Antibiotic Therapy}

Oral route. For low-risk adults only; use ciprofloxacin plus amoxicillin-clavulanate.

Monotherapy with vancomycin not indicated.

Received 7 December 2001; electronically published 13 February 2002

These guidelines were developed and issued on behalf of the Infectious Diseases Society of America.

Reprints or correspondence: Dr. Walter T. Hughes, St. Jude Children's Research Hospital, 332 North Lauderdale St., Memphis, TN 38105 (walter.hughes@ stjude.org).

Clinical Infectious Diseases 2002; 34:730-51

(C) 2002 by the Infectious Diseases Society of America. All rights reserved. $1058-4838 / 2002 / 3406-0002 \$ 03.00$
Choose therapy with 1 of the following agents: cefepime or ceftazidime, or imipenem or meropenem.

Two drugs without vancomycin. Choose an aminoglycoside plus antipseudomonal penicillin, cephalosporin (cefepime or ceftazidime), or carbapenem.

Vancomycin plus 1 or 2 antibiotics, if criteria for use of vancomycin are met. Choose cefepime or ceftazidime plus vancomycin, with or without an aminoglycoside; carbapenem plus vancomycin, with or without an aminoglycoside; or antipseudomonal penicillin plus an aminoglycoside and vancomycin.

\section{Modification of Therapy during the First Week of Treatment}

Patient becomes afebrile in 3-5 days. If an etiologic agent is identified, adjust therapy to the most appropriate $\operatorname{drug}(\mathrm{s})$. If no etiologic agent is identified and if the patient is at low risk initially, and oral antibiotic treatment was begun with no subsequent complications, continue use of the same drugs. If the patient was at low risk initially and therapy with intravenous drugs was begun with no subsequent complications, the regimen may be changed after $48 \mathrm{~h}$ to oral ciprofloxacin plus amoxicillin-clavulanate for adults or cefixime for children. If the patient is at high risk initially with no subsequent complications, continue use of the same intravenous drugs.

Persistent fever throughout the first 3-5 days. Reassess therapy on day 3. If there is no clinical worsening, continue use of the same antibiotics; stop vancomycin use if cultures do not yield organisms. If there is progressive disease, change antibiotics. If the patient is febrile after 5 days, consider adding an antifungal drug, with or without a change in antibiotic regimen. 


\section{Duration of Antibiotic Therapy}

Patient is afebrile by day 3. If the patient's neutrophil count is $\geqslant 500$ cells $/ \mathrm{mm}^{3}$ for 2 consecutive days, if there is no definite site of infection, and if cultures do not yield positive results, stop antibiotic therapy when the patient is afebrile for $\geqslant 48 \mathrm{~h}$. If the patient's neutrophil count is $<500$ cells $/ \mathrm{mm}^{3}$ by day 7 , if the patient was initially at low risk, and if there are no subsequent complications, stop therapy when the patient is afebrile for 5-7 days. If the patient was initially at high risk and there are no subsequent complications, continue antibiotic therapy.

Persistent fever on day 3. If the patient's neutrophil count is $\geqslant 500$ cells $/ \mathrm{mm}^{3}$, stop antibiotic therapy 4-5 days after the neutrophil count is $\geqslant 500$ cells $/ \mathrm{mm}^{3}$. If the patient's neutrophil count is $<500$ cells $/ \mathrm{mm}^{3}$, reassess and continue antibiotic therapy for 2 more weeks; reassess and consider stopping therapy if no disease site is found.

\section{Use of Antiviral Drugs}

Antiviral drugs are not recommended for routine use unless clinical or laboratory evidence of viral infection is evident.

\section{Granulocyte Transfusions}

Granulocyte transfusions are not recommended for routine use.

\section{Use of Colony-Stimulating Factors}

Use of colony-stimulating factors is not routine but should be considered in certain cases with predicted worsening of course.

\section{Antibiotic Prophylaxis for Afebrile Neutropenic Patients}

Use of antibiotic prophylaxis is not routine because of emerging antibiotic resistance, except for the use of trimethoprim-sulfamethoxazole to prevent Pneumocystis carinii pneumonitis. Antifungal prophylaxis with fluconazole and antiviral prophylaxis with acyclovir or ganciclovir are warranted for patients undergoing allogenic hematopoietic stem cell transplantation.

\section{INTRODUCTION}

This article, prepared by the IDSA Fever and Neutropenia Guidelines Panel, updates guidelines established a decade ago and revised in 1997 [1] by the IDSA for the use of antimicrobial agents to treat neutropenic patients with unexplained fever. The purpose is to assist internists, pediatricians, and family practitioners in the treatment of febrile neutropenic patients who have cancer and other underlying myelosuppressive diseases. The guidelines were prepared by a panel of experts in oncology and infectious diseases, peer-reviewed by an external group of knowledgeable practitioners, reviewed and approved by the Practice Guidelines Committee, and approved as published by the IDSA.
It is important to note that the guidelines are general and must be applied wisely with respect to variations in individual patients and types of infections, settings in which patients are being treated, antimicrobial susceptibility patterns, underlying causes of neutropenia, and expected time to recovery. The recommendations are based, whenever possible, on scientific publications and peer-reviewed information that has been formally presented at national and international meetings. When firm recommendations cannot be made, usually because of inadequate scientific data, the Guidelines Panel of the IDSA has offered suggestions based on the consensus of its members, all of whom have extensive experience in the treatment of neutropenic patients. These guidelines have been derived predominantly from knowledge of and experience with hematopoietic and lymphoproliferative malignancies, but they can be applied in general to febrile neutropenic patients with other neoplastic diseases.

Attempts have been made to estimate the validity of a particular recommendation or statement by use of the weighting system described in the 1997 guidelines (table 1) [1]. A ranking of A-E indicates the strength of this recommendation, and the Roman numerals I-III indicate the quality of evidence These rankings are presented in parentheses after specific recommendations.

We emphasize that no specific scheme, no specific drug or combination of drugs, and no specific period of treatment can be unequivocally applied to all febrile neutropenic patients. When possible, it is advisable to involve an infectious diseases specialist who is knowledgeable and interested in infections of the immunocompromised host.

Most of the information and recommendations made in the 23-page 1997 guidelines [1] are still valid. In an attempt to make the new guidelines more user-friendly, some of the background information and references from the 1997 version have not been included here. We have not addressed the management of drug-related allergies and other adverse effects from drugs because of limited data specific for neutropenic patients. The general principles of practice for nonneutropenic patients are also reasonable for neutropenic patients.

\section{CLINICAL FEATURES OF THE NEUTROPENIC HOST}

At least one-half of neutropenic patients who become febrile have an established or occult infection, and at least one-fifth of patients with neutrophil counts of $<100$ cells $/ \mathrm{mm}^{3}$ have bacteremia. The organisms that cause bacteremia are listed in table 2. Fungi are common causes of secondary infection among neutropenic patients who have received courses of broad-spectrum antibiotics and may also cause primary infections.

The primary anatomic sites of infection often include the alimentary tract, where cancer chemotherapy-induced mucosal damage allows invasion of opportunistic organisms. Similarly, 
Table 1. Infectious Diseases Society of America-United States Public Health Service Grading System for ranking recommendations in clinical guidelines.

\begin{tabular}{cl}
\hline Category, grade & \multicolumn{1}{c}{ Definition } \\
\hline $\begin{array}{c}\text { Strength of recommendation } \\
\text { A }\end{array}$ & Good evidence to support a recommendation for use \\
B & Moderate evidence to support a recommendation for use \\
C & Poor evidence to support a recommendation \\
D & Moderate evidence to support a recommendation against use \\
E & Good evidence to support a recommendation against use \\
Quality of evidence & \\
I & Evidence from $\geqslant 1$ properly randomized, controlled trial \\
II & Evidence from $\geqslant 1$ well-designed clinical trial, without randomiza- \\
& tion; from cohort or case-controlled analytic studies (preferably \\
& from $>1$ center); from multiple time-series; or from dramatic \\
III & results from uncontrolled experiments \\
& Evidence from opinions of respected authorities, based on clinical \\
experience, descriptive studies, or reports of expert committees
\end{tabular}

damage to the integument by invasive procedures, such as placement of vascular access devices, often provides portals of entry for infectious organisms.

\section{Definitions}

Fever. A temperature that is clearly greater than the normal constitutes a febrile state. In practice, a single oral temperature measurement of $\geqslant 38.3^{\circ} \mathrm{C}\left(101^{\circ} \mathrm{F}\right)$, in the absence of obvious environmental causes, is usually considered to be a fever. A temperature of $\geqslant 38.0^{\circ} \mathrm{C}\left(100.4^{\circ} \mathrm{F}\right)$ for $\geqslant 1 \mathrm{~h}$ indicates a febrile state.

Neutropenia. When the neutrophil count decreases to $<1000$ cells $/ \mathrm{mm}^{3}$, increased susceptibility to infection can be expected, with the frequency and severity inversely proportional to the neutrophil count [2-4]. Patients with neutrophil counts of $<500$ cells $/ \mathrm{mm}^{3}$ are at considerably greater risk for infection than are those with counts of $<1000$ cells $/ \mathrm{mm}^{3}$, and patients with counts of $\leqslant 100$ cells $/ \mathrm{mm}^{3}$ are at greater risk than are those with counts of $<500$ cells $/ \mathrm{mm}^{3}$. In addition to the number of circulating neutrophils, the duration of neutropenia is an important determinant of infection. A low nadir in the neutrophil count and protracted neutropenia (i.e., neutrophil count of $<500$ cells/ $\mathrm{mm}^{3}$ for 10 days) are major risk factors for impending infection $[2,5]$. In addition to quantitative changes in neutrophil counts, abnormalities of phagocytic function or other deficits in the immune response may further increase the risk for infection in a neutropenic host.

\section{EVALUATION}

Symptoms and signs of inflammation may be minimal or absent in the severely neutropenic patient, especially if accompanied by anemia [6]. Diminished or absent induration, erythema, and pustulation in response to bacterial infection leave the patient with a cutaneous infection without typical cellulitis, a pulmonary infection without discernible infiltrate on a radiograph, meningitis without pleocytosis in the CSF, and a urinary tract infection without pyuria. Nevertheless, a search should be undertaken for subtle symptoms and signs, including pain at the sites that are most commonly infected. These sites are the periodontium; the pharynx; the lower esophagus; the lung; the perineum, including the anus; the eye (fundus); and the skin, including bone marrow aspiration sites, vascular catheter access sites, and tissue around the nails.

Specimens should be obtained immediately for culture for bacteria and fungi. If a central venous access device is in place, some authorities, including the new "IDSA Guidelines for the Management of Intravascular Catheter-Related Infections" [7], recommend that $\geqslant 1$ set of blood samples be obtained for culture from the device lumen(s) as well as from a peripheral vein. Other investigators believe that culture only of a blood sample obtained from a central venous catheter is adequate $[8,9]$. Quantitative blood cultures, although not necessarily recommended routinely for all patients, may be helpful for those suspected of having a catheter-related infection, for whom specimens obtained from a central venous catheter and a peripheral vein should be compared $[7,10]$. High-grade bacteremia ( $>500$ $\mathrm{cfu} / \mathrm{mL}$ ) is associated with greater morbidity and mortality rates than is lower-grade bacteremia [11]. The yield of bacterial and fungal isolates is related to the culture systems used [12] and the volume of the blood sample [13]. Microbiology diagnostic laboratories must stay abreast of new technological developments related to the causative organisms unique to the neutropenic host. If a catheter entry site is inflamed or draining, the fluid exuded should be examined by Gram staining and culture for bacteria and fungi. If such lesions are persistent or 
chronic, stains and cultures for nontuberculous mycobacteria should be performed [14].

Little clinically useful information is gained from performing routine cultures of samples from the anterior nares, oropharynx, urine, and rectum, when lesions or disease processes are absent. However, for infection-control purposes, culture of anterior nasal samples can reveal colonization with methicillinresistant Staphylococcus aureus, penicillin-resistant pneumococci, or Aspergillus species, and culture of rectal samples can yield Pseudomonas aeruginosa, multidrug-resistant, gram-negative bacilli, or vancomycin-resistant enterococci. Such results may be useful collectively for infection control. Candida tropicalis in surveillance cultures has been associated with an increased risk for subsequent infection due to this fungus [15].

Diarrhea believed to be of infectious etiology should be evaluated according the "IDSA Practice Guidelines for the Management of Infectious Diarrhea" [16]. Culture of urine samples is indicated if signs or symptoms of urinary tract infection exist, a urinary catheter is in place, or the findings of urinalysis are abnormal. Examination of CSF specimens is not recommended as a routine procedure but should be considered if a CNS infection is suspected and thrombocytopenia is absent or manageable. Chest radiographs should be obtained whenever signs or symptoms of respiratory tract abnormality are present. Some experts recommend chest radiography for persons who are to be treated as outpatients, even without evidence of pulmonary infection. A baseline radiograph is helpful for neutropenic patients who subsequently develop respiratory symptoms or evidence of an infiltrate, but it is not cost-effective on a routine basis. Of note, high-resolution CT will reveal evidence of pneumonia in more than one-half of febrile neutropenic patients with normal findings on chest radiographs [17]. Aspiration or biopsy of skin lesions suspected of being infected should be performed for cytologic testing, Gram staining, and culture [18].

Complete blood cell counts and determination of the levels of serum creatinine and urea nitrogen are needed to plan supportive care and to monitor for the possible occurrence of drug toxicity. These tests should be done at least every 3 days during the course of intensive antibiotic therapy. The use of some drugs, such as amphotericin $\mathrm{B}$, will require more frequent measuring of creatinine as well as electrolyte levels. Monitoring of serum transaminase levels is advisable for patients with complicated courses or suspected hepatocellular injury. Levels of circulating C-reactive protein, IL-6, IL-8, and procalcitonin may be affected by bacteremia in neutropenic patients with fever [19-22] but the association is not sufficiently consistent to warrant their use in clinical practice.

Recommendations for evaluation. Initial evaluation should consist of a thorough physical examination; a complete blood cell count; measurement of serum levels of creatinine, urea nitrogen, and transaminases; and culture of blood samples (obtained from a peripheral vein and/or a catheter). A chest radiograph is indicated for patients with respiratory signs or symptoms or if outpatient management is planned (B-III).

\section{INITIAL ANTIBIOTIC THERAPY}

Because the progression of infection in neutropenic patients can be rapid, and because such patients with early bacterial infections cannot be reliably distinguished from noninfected patients at presentation, empirical antibiotic therapy should be administered promptly to all neutropenic patients at the onset of fever (figure 1). Afebrile patients who are neutropenic but who have signs or symptoms compatible with an infection should also have empirical antibiotic therapy begun in the same manner as do febrile patients.

Gram-positive bacteria (table 2 ) now account for $\sim 60 \%-70 \%$ of microbiologically documented infections, although the rate of gram-negative infections is increasing in some centers. Some of the gram-positive organisms may be methicillin resistant and, therefore, are susceptible only to vancomycin, teicoplanin (which is not currently available in the United States), quinupristin-dalfopristin, and linezolid. These are often more indolent infections (e.g., infections due to coagulase-negative staphylococci, vancomycin-resistant enterococci, or Corynebacterium jeikeium), and a few days' delay in administration of specific therapy may not be detrimental to the patient's outcome, although it may prolong the duration of hospitalization. Other gram-positive bacteria ( $S$. aureus, viridans streptococci, and pneumococci) may cause fulminant infections resulting in serious complications or death, if not treated promptly [23, 24]. Gram-negative bacilli, especially P. aeruginosa, Escherichia coli, and Klebsiella species (table 2), remain prominent causes of infection and must be treated with selected antibiotics [25-29]. Although fungal infections are usually superinfections, in some cases, Candida species or other fungi can cause primary infections.

In the selection of the initial antibiotic regimen, one should consider the type, frequency of occurrence, and antibiotic susceptibility of bacterial isolates recovered from other patients at the same hospital. The use of certain antibiotics may be limited by special circumstances, such as drug allergy or organ (e.g., renal or hepatic) dysfunction. Such drugs as cisplatin, amphotericin B, cyclosporine, vancomycin, and aminoglycosides should be avoided in combination, if possible, because of their additive renal toxicity [30]. Drug plasma concentrations should be monitored when they are helpful in predicting therapeutic success and toxicity (e.g., aminoglycosides).

Vascular access devices (e.g., Hickman-Broviac catheters or subcutaneous ports) may be left in place during antibiotic treat- 
Table 2. Bacterial causes of febrile episodes in neutropenic patients.

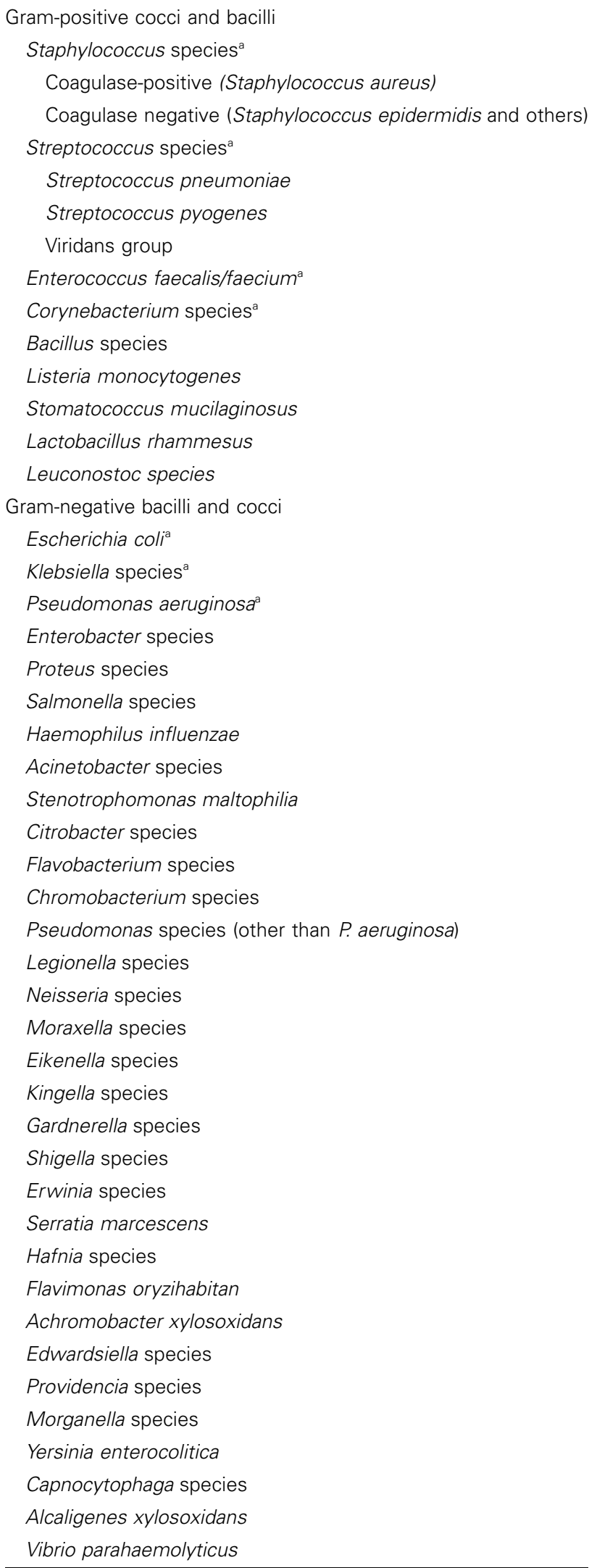

(continued)
Table 2. (Continued.)

\begin{tabular}{l} 
Chryseobacterium meningosepticum \\
Burkholderia cepacia \\
Fusobacterium nucleatum \\
Leptotrichia buccalis \\
Methylobacterium species \\
Anaerobic cocci and bacilli \\
Bacteroides species \\
Clostridium species \\
Fusobacterium species \\
Propionibacterium species \\
Peptococcus species \\
Veillonella species \\
Peptostreptococcus species \\
\hline a The most common causes of bacteremia.
\end{tabular}

ment for most patients, even if infection of a local entry site or catheter-related bacteremia is detected (A-II). S. aureus and coagulase-negative staphylococci are the most common causes of catheter-associated infections [31,32], and these often respond to treatment with parenteral antibiotics without removal of the catheter, unless a tunnel infection has become established (B-II) [31]. Response to antibiotic therapy alone is most likely and complications are least likely with coagulase-negative staphylococcal catheter-related infections, but catheter removal may be required for cure, regardless of the etiology, if the infection is recurrent or response to antibiotics is not apparent after 2 or 3 days of therapy. Evidence of a subcutaneous tunnel or periport infection, septic emboli, hypotension associated with catheter use, or a nonpatent catheter are indications for removal, along with prompt administration of antibiotics (AII). Catheter removal combined with generous debridement of infected tissue is also advisable for patients with atypical mycobacterial infection (A-II) [33]. Bacteremia due to Bacillus species, $P$. aeruginosa, Stenotrophomonas maltophilia, C. jeikeium, or vancomycin-resistant enterococci, and fungemia due to Candida species [7], often respond poorly to antimicrobial treatment, and prompt removal of the catheter is recommended, if possible (CIII). Established infections with Acinetobacter species also often require removal of the infected catheter.

The use of antibiotic-impregnated catheters, administration of antibiotics through each lumen of the involved catheter, rotation of antibiotic delivery through multilumen catheters, and the use antibiotic-containing heparin lock solutions ("antibiotic lock therapy") to supplement systemic therapy have been proposed by some investigators. Such practices are controversial, and none can be recommended as a standard of practice for all patients. For selected application, the reader is referred to the 2001 guidelines for the management of intravascular catheter-related infections, which were developed jointly by 


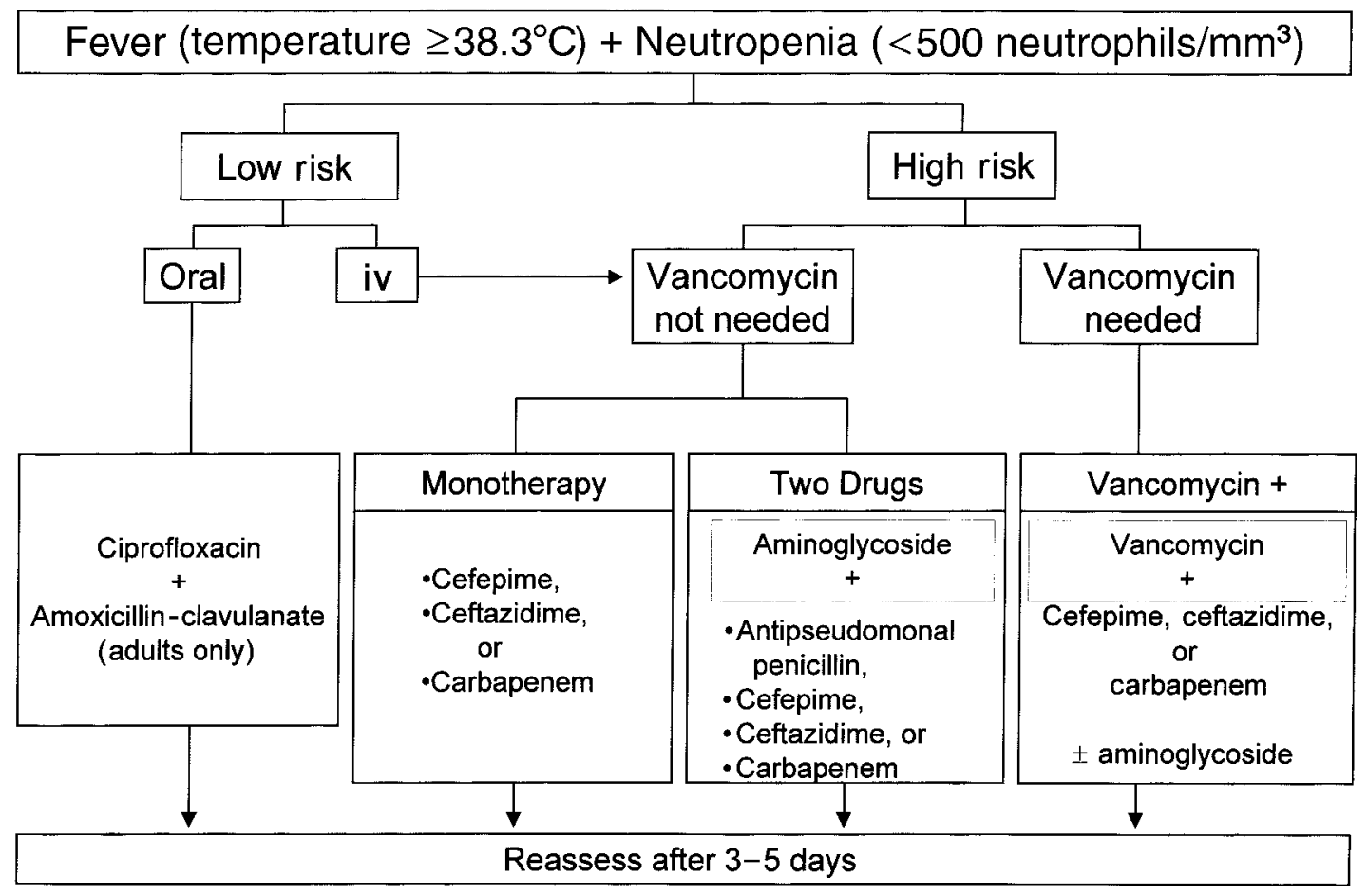

Figure 1. Algorithm for initial management of febrile neutropenic patients. See tables 3 and 4 for rating system for patients at low risk. Carbapenem, imipenem or meropenem.

IDSA, the American College of Critical Care Medicine, and the Society for Healthcare Epidemiology of America [7]. Vancomycin should not routinely be used prophylactically in catheters. Insufficient data are available to recommend the routine supplemental use of urokinase for management of catheterrelated infection.

Despite extensive clinical studies since the 1970s, no single empirical therapeutic regimen for the initial treatment of febrile patients with neutropenia can be recommended. The results from study to study are often not comparable, because the definitions of infectious diseases and the criteria used to assess the response to therapy vary considerably $[34,35]$. Although it is generally agreed that many antibiotic regimens are effective in the control of infection with minimal toxicity, careful selection based on local patterns of infection and antibiotic susceptibilities may enhance efficacy while maintaining safety and minimizing costs. For example, several studies have indicated that not all $\beta$-lactam antibiotics are equally effective, at least at certain institutions. Antibiotic resistance among gram-negative bacilli may limit the efficiency of some $\beta$-lactams at some institutions [36-38].

\section{Level of Risk for Oral Antibiotics and Outpatient Management}

Treatment of carefully selected febrile neutropenic patients with oral antibiotics alone appears to be feasible for adults at low risk for complications (A-I) [39-53]. In general, the use of antibiotics by the oral route may be considered only for patients who have no focus of bacterial infection or symptoms and signs suggesting systemic infection (e.g., rigors, hypotension) other than fever. Some patients may reliably receive their prescribed therapy as outpatients, although many of the studies that have supported treatment with oral antibiotics involved hospitalized patients [43, 45]. Vigilant observation and prompt access to appropriate medical care must also be ensured $24 \mathrm{~h}$ per day, 7 days per week. For many patients and some institutions, outpatient therapy may not be advisable. Patients with recovering phagocyte counts are generally considered to be better candidates for outpatient treatment than are patients with decreasing counts or no indication of marrow recovery.

Factors favoring low risk for serious infections among febrile neutropenic patients, which have been identified in controlled studies, are listed in table 3 [4, 42-53]. These characteristics may serve as guidelines for the selection of patients for outpatient therapy. A recent international collaborative study of 1139 febrile and neutropenic patients with malignancy established and validated a scoring system to identify, at the time of presentation with fever, those patients with low risk for complications, including mortality [50]. Factors associated with lower risk for complications and a higher rate of favorable outcome $(P<.001)$ were as follows: age $<60$ years (children not included), cancer in partial or complete remission, no symptoms or only mild to moderate symptoms of illness, outpatient 
Table 3. Factors that favor a low risk for severe infection among patients with neutropenia.

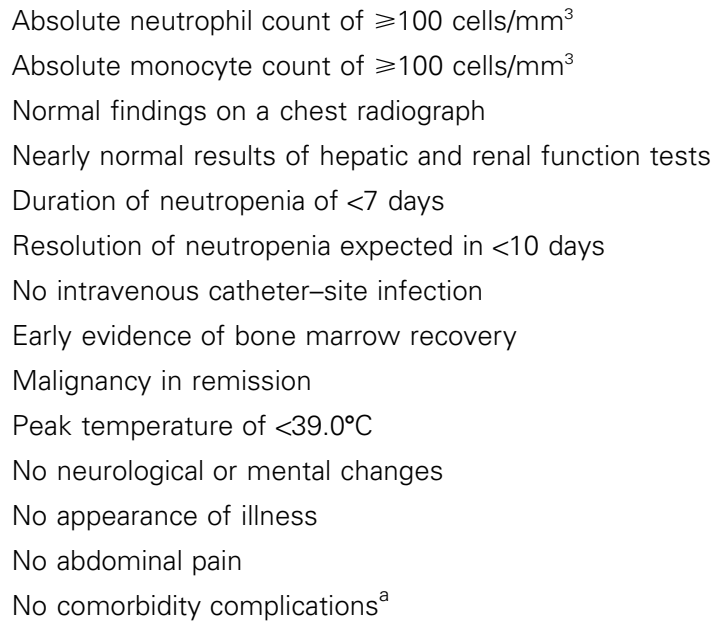

NOTE. Data are adapted from [4, 42-49, 51-53].

a Concomitant condition of significance (e.g., shock, hypoxia, pneumonia or other deep-organ infection, vomiting, or diarrhea).

status at the time of onset of fever, temperature $<39.0^{\circ} \mathrm{C}$, normal findings on chest radiographs, absence of hypotension, respiratory rate of $\leqslant 24$ breaths/min, absence of chronic pulmonary diseases, absence of diabetes mellitus, absence of confusion or other signs of mental status alteration, absence of blood loss, absence of dehydration, no history of fungal infection, and no receipt of antifungal therapy during the 6 months before presentation with fever. Integer weights were assigned for 7 characteristics to develop a risk-index score (table 4), which was subsequently tested for validation. A risk-index score of $\geqslant 21$ identified low-risk patients with a positive predictive value of $91 \%$, specificity of $68 \%$, and sensitivity of $71 \%$. This study generally supports the earlier studies of Talcott et al [51]. The database for this system did not include infants and children.

Recently, Klaassen et al. [46] prospectively derived and validated a clinical prediction rule for pediatric oncology patients with fever and neutropenia. Children presenting with an initial absolute monocyte count of $\geqslant 100$ cells $/ \mathrm{mm}^{3}$, with no comorbidity, and with a normal chest radiograph findings are at lowest risk for significant bacterial infections.

As an alternative to initial outpatient therapy, early discharge with continued outpatient therapy for selected patients may be considered after a brief inpatient admission during which intravenous therapy is initiated, fulminant infection is excluded, and the status of initial culture specimens is ascertained [52, $53]$.

\section{Treatment with Intravenous Antibiotics}

The first step in antibiotic selection is to decide whether the patient is a candidate for inpatient or outpatient management with oral or intravenous antibiotics (figure 1).
Three general schemes of intravenous antibiotic therapies with similar efficacy are considered here, with the caveat that one may be more appropriate for certain patients and in certain institutions than others. The schemes are as follows: singledrug therapy (monotherapy), 2-drug therapy without a glycopeptide (vancomycin), and therapy with glycopeptide (vancomycin) plus 1 or 2 drugs.

Single-drug therapy (monotherapy). Several studies have shown no striking differences between monotherapy and multidrug combinations for empirical treatment of uncomplicated episodes of fever in neutropenic patients (A-I) [54-71]. A thirdor fourth-generation cephalosporin (ceftazidime or cefepime) or a carbapenem (imipenem-cilastatin or meropenem) may be used successfully as monotherapy $[68,72,73-78]$. Physicians should be aware that extended-spectrum $\beta$-lactamases and type $1 \beta$-lactamases have reduced the utility of ceftazidime for monotherapy [36]. Cefepime, imipenem-cilastatin, and meropenem, unlike ceftazidime, have excellent activity against viridans streptococci and pneumococci. Vancomycin was shown to be required less frequently with cefepime than with ceftazidime monotherapy [75]. A prospective double-blind study of 411 patients who had cancer showed that the rate of clinical response was higher in febrile neutropenic patients treated with meropenem than it was in those treated with ceftazidime [74]. Elsewhere, similar results have been observed [76]. Piperacillintazobactam has also been found to be effective as monotherapy, but its use has not been studied as extensively as that of the other agents $[64,65]$.

The patient must be monitored closely for nonresponse, emergence of secondary infections, adverse effects, and the development of drug-resistant organisms. Addition of other anti-

Table 4. Scoring index for identification of low-risk febrile neutropenic patients at time of presentation with fever.

\begin{tabular}{lc}
\hline Characteristic & Score \\
\hline Extent of illness $^{\text {a }}$ & \\
No symptoms & 5 \\
Mild symptoms & 5 \\
Moderate symptoms & 3 \\
No hypotension & 5 \\
No chronic obstructive pulmonary disease & 4 \\
Solid tumor or no fungal infection & 4 \\
No dehydration & 3 \\
Outpatient at onset of fever & 3 \\
Age $<60$ years & \\
\hline
\end{tabular}

NOTE. Highest theoretical score is 26 . A risk index score of $\geqslant 21$ indicates that the patient is likely to be at low risk for complications and morbidity. The scoring system is derived from [50].

${ }^{a}$ Choose 1 item only.

b Does not apply to patients $\leqslant 16$ years of age. Initial monocyte count of $\geqslant 100$ cells $/ \mathrm{mm}^{3}$, no comorbidity, and normal chest radiograph findings indicate children at low risk for significant bacterial infections [46]. 
biotics may be necessary as the clinical course progresses. In particular, the spectrum of these drugs does not usually cover coagulase-negative staphylococci, methicillin-resistant S. aureus, vancomycin-resistant enterococci, some strains of penicillin-resistant Streptococcus pneumoniae, and viridans streptococci. Cefepime or ceftazidime may be used in the presence of mild or moderate renal dysfunction without dose modification and for patients being treated with nephrotoxic drugs, such as cisplatin, cyclosporin, or amphotericin B.

Quinolones, such as ciprofloxacin, also have been evaluated for use as monotherapy in limited studies showing both favorable [77-79] and unfavorable results [80, 81]. The general use of quinolones for prophylaxis among afebrile neutropenic patients limits this class of drugs for initial therapy. Currently, in comparison with the other antibiotics recommended for intravenous monotherapy, quinolones cannot be recommended for routine initial intravenous monotherapy.

Treatment with aminoglycosides alone is suboptimal, even though the bacterial isolate may be susceptible in vitro.

Two-drug therapy without a glycopeptide antibiotic (vancomycin). The most commonly used 2-drug therapy, excluding regimens with vancomycin, includes an aminoglycoside (gentamicin, tobramycin, or amikacin) with an antipseudomonal carboxypenicillin or ureidopenicillin (ticarcillin-clavulanic acid or piperacillin-tazobactam); an aminoglycoside with an antipseudomonal cephalosporin, such as cefepime or ceftazidime; and an aminoglycoside plus a carbapenem (imipenem-cilastatin or meropenem). Pertinent publications summarized in the 1997 guidelines [1] show that, generally, the different 2-drug combinations yield similar results when variations in experimental design, definitions, end points, and underlying primary diseases are taken into consideration. It is important to note, in reviewing older studies, that the susceptibility of isolates to antibiotics used at the time of the study may be different from susceptibilities of some bacterial isolates today.

Advantages of combination therapy are potential synergistic effects against some gram-negative bacilli [82] and minimal emergence of drug-resistant strains during treatment [83, 84]. The major disadvantages are the lack of activity of these combinations, such as ceftazidime plus an aminoglycoside, against some gram-positive bacteria, and the nephrotoxicity, ototoxicity, and hypokalemia associated with aminoglycoside compounds and carboxypenicillins. Limited studies show that a single daily dose of an aminoglycoside with ceftriaxone is as effective as multiple daily doses of these drugs [85] and as effective as monotherapy with ceftazidime [54], but data are insufficient to establish a practice standard. Serum levels of the aminoglycoside should be monitored as needed in patients with impaired renal function, and dosages should be adjusted until optimal therapeutic concentrations are achieved.
Quinolone-based combinations with $\beta$-lactams or glycopeptides are an option for initial therapy for patients not receiving quinolone prophylaxis. Newer agents (gatifloxacin, moxifloxacin, and levofloxacin) have been used selectively to treat patients who have cancer, but their roles will need to be evaluated further before recommendations can be made [86-89]. A recent large comparative trial showed that ciprofloxacin plus piperacillin-tazobactam is as effective as tobramycin and piperacillintazobactam [90]. Any initial antibiotic regimen should include drugs with antipseudomonal activity [91].

Therapy with glycopeptide (vancomycin) plus 1 or 2 drugs. Because of the emergence of vancomycin-resistant organisms, especially enterococci, associated with excessive use of vancomycin in the hospital, administration of vancomycin should be limited to specific indications. Hospitals should adopt the recommendations of the Hospital Infection Control Practices Advisory Committee of the Centers for Disease Control and Prevention (CDC) for preventing the spread of vancomycin resistance [92]. The European Organization for Research and Treatment of Cancer (EORTC)-National Cancer Institute of Canada study showed that vancomycin is not in general a necessary part of initial empirical antibiotic therapy, if it is available for subsequent treatment modifications [30, 93]. At institutions at which these infections are rare, vancomycin should be routinely withheld until the results of cultures indicate the need for this antibiotic.

Infections caused by gram-positive bacteria are frequently indolent, but some may be susceptible only to vancomycin and can, on occasion, be serious, leading to death in $<24 \mathrm{~h}$ if not promptly treated. Although vancomycin has not been shown to influence overall mortality due to gram-positive cocci as a group, mortality due to viridans streptococci may be higher among patients not initially treated with vancomycin $[94,95]$. Some strains of viridans streptococci are resistant to or tolerant of penicillin, but such antibiotics as ticarcillin, piperacillin, cefepime (but not ceftazidime), and carbapenems all have excellent activity against most strains. At institutions at which these gram-positive bacteria are common causes of serious infections, vancomycin may be incorporated into initial therapeutic regimens of some high-risk patients but discontinued 24-48 h later if no such infection is identified. Some organisms, such as Bacillus species and C. jeikeium, are susceptible only to vancomycin, but these infections are usually not severe. Inclusion of vancomycin in initial empirical therapy may be prudent for selected patients with the following clinical findings: (1) clinically suspected serious catheter-related infections (e.g., bacteremia, cellulitis), (2) known colonization with penicillin- and cephalosporin-resistant pneumococci or methicillin-resistant $S$. aureus, (3) positive results of blood culture for gram-positive bacteria before final identification and susceptibility testing, or 
(4) hypotension or other evidence of cardiovascular impairment [92].

For some physicians in some medical centers, intensive chemotherapy that produces substantial mucosal damage (e.g., high-dose cytarabine) or increases the risk for penicillin-resistant streptococcal infections (e.g., infection with viridans streptococci), as well as prophylaxis with quinolones for afebrile neutropenic patients before onset of fever, are also considered indications for vancomycin to be included in the initial regimen. Sudden increase of temperature to $>40^{\circ} \mathrm{C}$ has, to some extent, been predictive of sepsis with viridans streptococci [95].

Several studies have evaluated vancomycin drug combinations for the treatment of neutropenic patients with fever; these combinations have included vancomycin plus any of the following antibiotics: imipenem [72, 96], cefepime [60], amikacin and ticarcillin [94], meropenem [74], ciprofloxacin [97], aztreonam [98, 99], ceftazidime [100-104], ceftazidime and amikacin $[30,103]$, tobramycin and piperacillin $[105,106]$, ticarcillin [93], and ceftazidime and ticarcillin [38]. Although the combination of ceftazidime and vancomycin has been used most extensively in the past, in some medical centers, the possible risk of emergence of resistance to ceftazidime may justify the recommendation that vancomycin be preferentially used in combination with cefepime or a carbapenem (imipenem-cilastatin or meropenem).

Teicoplanin has been evaluated as an alternative to vancomycin (see appendix 1 in the 1997 guidelines [1]) in limited clinical trials $[105,106]$, but the drug has not received approval from the US Food and Drug Administration (FDA), and additional studies are needed to place it in proper perspective.

Linezolid, the first FDA-approved oxazolidinone, offers promise for treatment of drug-susceptible and -resistant gram-positive bacterial infections, including those due to vancomycin-resistant enterococci, although an associated myelosuppression may be problematic [107]. Quinupristin-dalfopristin, another drug that has recently been approved by the FDA, is also effective against vancomycin-resistant Enterococcus faecium. Further studies are needed before guideline recommendations can be made for these drugs.

\section{Treatment with Oral Antibiotics}

Several studies have evaluated oral absorbable broad-spectrum antibiotics for use as initial empirical therapy for febrile, neutropenic patients considered to be at low risk for bacterial infection [39-53]. The outcomes for low-risk patients treated with oral antibiotic therapy are generally equivalent to those for similar patients treated with intravenous antibiotic therapy when both treatment groups are managed in an inpatient setting in controlled studies $[43,45]$. Oral therapy has the advantages of reduced cost, facilitation of outpatient management, and avoidance of catheter use, thereby reducing the possibility of hospital- and catheter-related infection. Among the oral regimens that have been most thoroughly evaluated are ofloxacin, ciprofloxacin, and ciprofloxacin plus amoxicillin-clavulanate. Quinolones are generally not as effective as cephalosporins or carbapenems in treating gram-positive infections and may predispose to the development of viridans streptococcal sepsis [95]. Quinolones are not currently approved for use in children $<18$ years of age, although toxicity has not been high in pediatric trials [101]. Although controlled studies of children have assessed early switching from intravenous to oral antibiotics, other than quinolones $[52,53]$, only a few children have been included in studies of initial empirical oral therapy for febrile neutropenia [108]. At this time, there are insufficient data to recommend initial empirical oral therapy for fever and neutropenia in children. However, early discharge of patients while they are receiving treatment with cefixime after $\geqslant 48 \mathrm{~h}$ of inhospital observation of treatment with intravenous antibiotics may be considered for selected children $[52,53]$.

\section{Recommendations for Initial Regimen}

Figure 1 shows an algorithm for initial management. First, determine whether the patient is at low or high risk for serious life-threatening infection on the basis of the criteria observed at the time of presentation, which are presented in tables 3 and 4. If the risk is high, intravenous antibiotics must be used; if risk is low, the patient may be treated with either intravenous or oral antibiotics (A-II). Second, decide whether the patient qualifies for vancomycin therapy. If the patient qualifies, begin treatment with a 2- or 3-drug combination with vancomycin plus cefepime, ceftazidime, or a carbapenem, with or without an aminoglycoside. If vancomycin is not indicated, begin monotherapy with a cephalosporin (cefepime or ceftazidime) or a carbapenem (meropenem or imipenem-cilastatin) administered intravenously for uncomplicated cases. Two-drug combinations may be used for management of complicated cases or if antimicrobial resistance is a problem. Adults selected for oral therapy may receive ciprofloxacin plus amoxicillinclavulanate. Selection of patients for outpatient therapy must be done carefully from the low-risk group, depending on the capabilities of the medical center and doctor-patient relationship. Initial therapy with oral antibiotics alone is not recommended for children. Use current antibiotic susceptibility patterns from your local hospital laboratory as an aid in antibiotic selection.

\section{MANAGEMENT OF THE ANTIBIOTIC REGIMEN DURING THE FIRST WEEK OF THERAPY}

Receipt of antibiotic treatment for at least 3-5 days is usually required to determine efficacy of the initial regimen. From this point, decisions regarding further treatment are made on the 
basis of whether the patient had bacteremia or pneumonia, whether the fever has resolved, and whether the patient's condition has deteriorated. Some patients' conditions may deteriorate rapidly in $<3$ days, necessitating reassessment of the empirical regimen.

In several studies, the times to defervescence for febrile neutropenic patients with cancer who receive antibiotic regimens including cefepime, ceftazidime, ciprofloxacin, imipenem, and piperacillin (with or without aminoglycosides) are 2-7 days (median time, 5 days) [30, 85, 104, 109-111]. In a recent analysis of 488 episodes of fever and neutropenia, the median time to clinical response was 5-7 days [112]. The median time to defervescence among low-risk patients has been 2 days [43, 45], compared with 5-7 days for high-risk patients. When possible, despite the fact that the patient remains febrile, the clinician may wait 5 days to make any changes in antimicrobial regimen, unless a change is mandated by clinical deterioration or the results of a new culture.

\section{Patient is Afebrile within 3-5 Days of Treatment}

If a causative microbe is identified, the antibiotic regimen may be changed, if necessary, to provide optimal treatment with minimal adverse effects and lowest cost, but broad-spectrum coverage should be maintained to prevent breakthrough bacteremia. Antibiotic treatment should be continued for a minimum of 7 days or until culture results indicate that the causative organism has been eradicated, infection at all sites has resolved, and the patient is free of significant symptoms and signs (figure 2). It is desirable for the neutrophil count to be $\geqslant 500$ cells $/ \mathrm{mm}^{3}$ before treatment is stopped. However, if the neutropenia is prolonged and the aforementioned responses have been achieved, consideration can be given to discontinuation of treatment before a neutrophil count of $\geqslant 500$ cells/ $\mathrm{mm}^{3}$ is reached. This approach can be taken if the patient can be carefully observed, the mucous membranes and integument are intact (e.g., no mucositis, ulcerations, evidence of catheter site infection, or bleeding sites are present), and no invasive procedures or ablative chemotherapy are impending.

In the absence of discernible infectious disease (e.g., pneumonitis, enterocolitis, cecitis, endocarditis, catheter-associated infection, or severe cellulitis) and of positive culture results, treatment for compliant adults may be changed after $\geqslant 2$ days of intravenous therapy to an oral antibiotic combination of ciprofloxacin and amoxicillin-clavulanic acid [43, 45]. Among children, 2 studies $[52,53]$ show that a change after $48-72 \mathrm{~h}$ of intravenous antibiotics to oral cefixime alone provides therapy that is as effective and safe as continuation of intravenous antibiotics. Children who lack signs of sepsis (chills, hypotension, and requirement for fluid resuscitation) and severe mucositis at the time of admission and throughout their courses, who are afebrile for $\geqslant 48 \mathrm{~h}$, who have neutrophil counts of $\geqslant 100$ cells $/ \mathrm{mm}^{3}$, and who are at low risk for complications may have intravenous antibiotic treatment stopped and therapy continued with oral cefixime. The controlled studies of adults $[43,45]$ and children $[52,53]$ were done with inpatients, so one cannot be assured that similar results will occur if patients are discharged while receiving treatment with oral antibiotics.

Some investigators have advocated discontinuation of antibiotic therapy for patients without documented infections and with signs of early marrow recovery before completing a 7-day course [113-116]. At present, there is not enough evidence or experience with this approach to endorse its use.

It is important to realize that the suggestions made here are somewhat arbitrary, and a comprehensive assessment is essential for each patient. Antibiotic therapy alone, in the presence of persistent neutropenia, may suppress but not eradicate the infection.

Recommendations for afebrile patients. Figure 2 presents a guide for treatment of patients who become afebrile within 3-5 days of starting treatment. Modify antibiotic therapy for specific organisms, if identified, and continue use of broadspectrum antibiotics for $\geqslant 7$ days, until cultures are sterile and the patient has clinically recovered. If the causative organism is not found and the patient is receiving drugs intravenously and was at low risk at the onset of treatment, treatment may be changed to oral ciprofloxacin plus amoxicillin-clavulanate for adults or cefixime for children after $48 \mathrm{~h}$, if clinically preferable. The same intravenous antibiotics should be continued for high-risk patients (B-II).

\section{Persistent Fever throughout the First 3-5 Days of Treatment}

Fever that persists for $>3$ days in patients for whom no infected site or organism has been identified suggests that the patient has a nonbacterial infection, a bacterial infection resistant to the antibiotic(s) or slow to respond to the drug in use, the emergence of a second infection, inadequate serum and tissue levels of the antibiotic(s), drug fever, cell wall-deficient bacteremia [117], or infection at an avascular site (e.g., "abscesses" or catheters). In reassessing the patient's condition after 3 days of treatment, the physician should attempt to identify factor(s) that might account for nonresponsiveness (figure 3). However, some patients with microbiologically defined bacterial infections, even when adequately treated, may require $\geqslant 5$ days of therapy before defervescence occurs [30, 85, 104, 111, 112].

Reassessment includes a review of all previous culture results, a meticulous physical examination, chest radiography, ascertaining the status of vascular catheters, culturing of additional blood samples and specimens of specific sites of infection, and diagnostic imaging of any organ suspected of having infection. If possible, the determination of serum concentrations of antibiotics, especially aminoglycosides, may be useful in assessment of drug therapy. Ultrasonography and high-resolution CT 


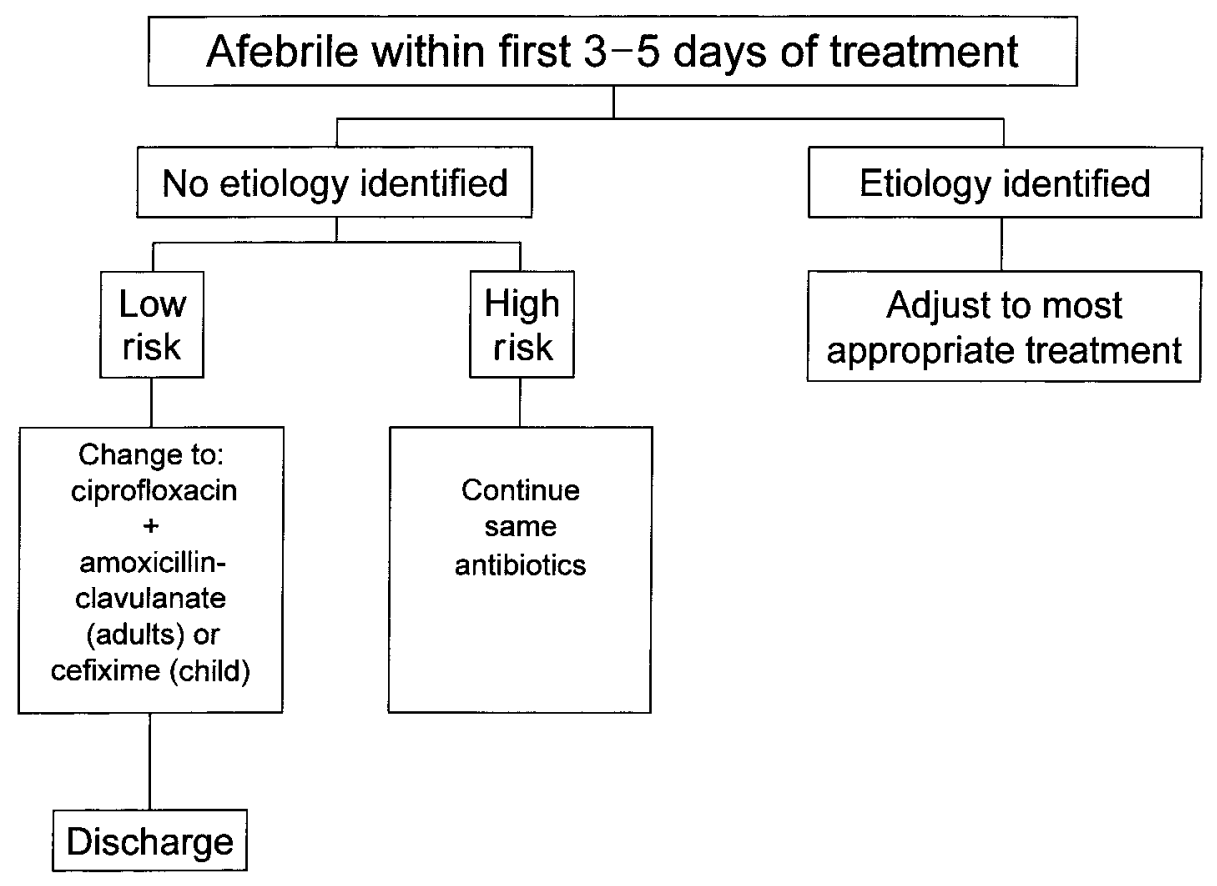

Figure 2. Guide for management of patients who become afebrile in the first $3-5$ days of initial antibiotic therapy. See tables 3 and 4 for rating system for patients at low risk.

may be helpful, especially for patients with pneumonitis, sinusitis, and cecitis. Additional studies may be done to identify relatively uncommon causes of fever. If reassessment yields a cause of the fever or strongly suggests a cause not adequately covered by the initial antibiotic regimen, a change should be made accordingly.

If the fever persists after 5 days of antibiotic therapy and reassessment does not yield a cause, 1 of 3 choices of management should be made (figure 3): (1) continue treatment with the initial antibiotic(s), (2) change or add antibiotic(s), (3) or add an antifungal drug (amphotericin B) to the regimen, with or without changing the antibiotics. A fourth choice-withdrawal of all antimicrobial drugs - will not be discussed as a valid option in these general guidelines, although, in some highly individualized cases (such as cases in which the fever is thought to be of noninfectious origin), physicians may elect to stop antibiotic therapy.

If no discernible changes in the patient's condition have occurred (i.e., the patient remains febrile but stable) during the first 4-5 days of initial antibiotic treatment, and if reevaluation yields no new information to the contrary, the initial antibiotic regimen can be continued. This decision will be strengthened if the neutropenia can be expected to resolve within the ensuing 5 days.

If evidence of progressive disease or complication (such as the onset of abdominal pain due to enterocolitis or cecitis, new or worsening mucous membrane lesions, drainage or reactions around catheter entry and/or exit sites, pulmonary infiltrates, toxicity or other adverse effects caused by the drugs, or changes in the bacteria in the mucous membranes [e.g., acquisition of $P$. aeruginosa after admission samples for culture were taken]) becomes apparent during the initial antibiotic course, consideration should be given to either the addition of appropriate antibiotics or a change to different antibiotics. Whether a change is indicated will also depend on the initial antibiotic regimen.

If the initial antibiotic regimen is monotherapy or 2-drug therapy without vancomycin, vancomycin may be considered if any of the criteria for use of vancomycin mentioned above have occurred (C-III). If a blood- or site-specific organism is isolated, the most appropriate antibiotic should be used while continuing broad-spectrum coverage.

If the initial treatment included vancomycin as a part of the therapeutic regimen, consideration should be given to the withdrawal of vancomycin to minimize the development of antibacterial resistance to this important drug. By day 3 , the results of admission cultures will be available to support a decision to stop vancomycin therapy. The other initial antibiotics may be continued if there is no evidence of disease progression, or, if the patient is in a low-risk category (figure 2), an oral antibiotic may be given, even if the patient is febrile (C-III).

The third choice to consider is the addition of antifungal therapy. Amphotericin B is usually the drug of choice. Studies in 1982 [118] and 1989 [119] suggested that up to one-third of febrile neutropenic patients who do not respond to a 1-week course of antibiotic therapy have systemic fungal infections that, in most cases, are caused by Candida or Aspergillus species. Although clinicians disagree as to when, and even if, ampho- 


\section{Persistent fever during first 3-5 days of treatment: no etiology}

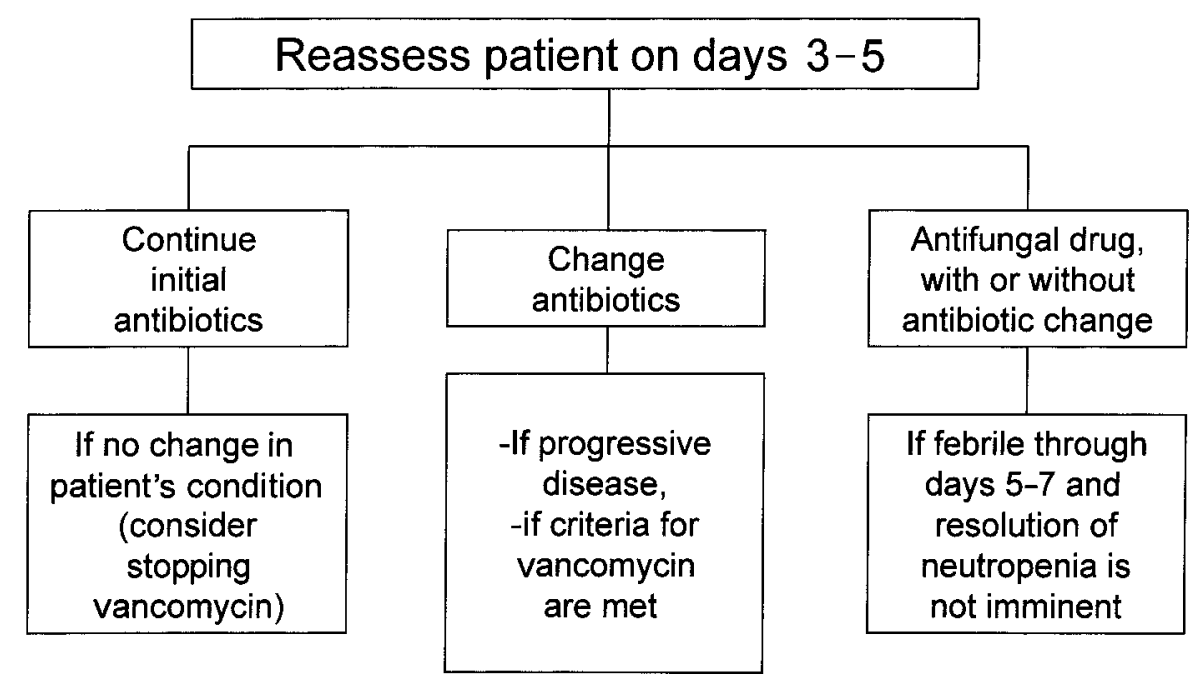

Figure 3. Guide to treatment of patients who have persistent fever after $3-5$ days of treatment and for whom the cause of the fever is not found.

tericin B therapy should be introduced empirically, most believe that the patient who remains febrile and profoundly neutropenic for $\geqslant 5$ days, despite the administration of broad-spectrum antibiotics in adequate dosages, is a candidate for antifungal therapy. Individual cases may have clinical features that will direct use of amphotericin B earlier, later, or not at all. Such an exception might be the patient who has no discernible fungal lesion, has neither Candida nor Aspergillus species isolated from any site, and is expected to have an increase in the neutrophil count within a few days. In this case, treatment with amphotericin B could be withheld and the patient should be monitored carefully, if the patient is clinically stable. Every effort should be made to determine whether systemic fungal infection exists (e.g., biopsy of lesions should be performed; radiographs of chest and sinuses should be obtained; nasal endoscopy should be performed, if indicated, to investigate sinusitis; and cultures and CT of the abdomen and chest should be done) before amphotericin B therapy is started. The empirical decision to start use of the drug is not as difficult as the decision to discontinue use of the drug. Much of the evaluation at this time is to aid in a decision about when to stop antifungal treatment later. It is noteworthy that a CT scan done after the neutrophil count has recovered may show some enhancement of earlier infection even with successful treatment.

Comparative trials have indicated that lipid formulations of amphotericin B can be used as alternatives to amphotericin B deoxycholate for empirical therapy. Although they do not appear to be substantially more effective, there is less drug-related toxicity [119-122]. For example, in a clinical trial comparing amphotericin B with liposomal amphotericin B (AmBisome; Fujisawa Healthcare), the overall success rates were the same for both preparations, although breakthrough fungal infections occurred more frequently with amphotericin B and there was a higher proportion of side effects with this preparation [120]. A double-blind comparative study showed that liposomal amphotericin B (AmBisome) had superior safety to amphotericin B lipid complex (Abelcet; Elan Pharmaceuticals) and a similar therapeutic success rate [121]. The investigators of a comparative unblinded study concluded that Abelcet and AmBisome are equally effective for the treatment of suspected and documented fungal infections in patients with leukemia; safety related to severe adverse effects on the kidneys or liver was similar, but the milder toxic reactions associated with acute infusion-related reactions and increases in the creatinine level were associated with Abelcet use, and more liver function test abnormalities were associated with AmBisome use [122].

Two recent prospective randomized trials have demonstrated that fluconazole is an acceptable alternative to amphotericin B for use as empirical antifungal therapy at institutions at which mold infections (e.g., Aspergillus species) and drug-resistant Candida species (Candida krusei and some strains of Candida glabrata) are uncommon. Patients should not be considered for empirical fluconazole treatment if they have symptoms of sinusitis or radiographic evidence of pulmonary infection or have received fluconazole as prophylaxis. Also, any patient from whom Aspergillus species were yielded on culture should not be considered. Patients with pulmonary infection or sinusitis have a high probability of infection with Aspergillus species or 


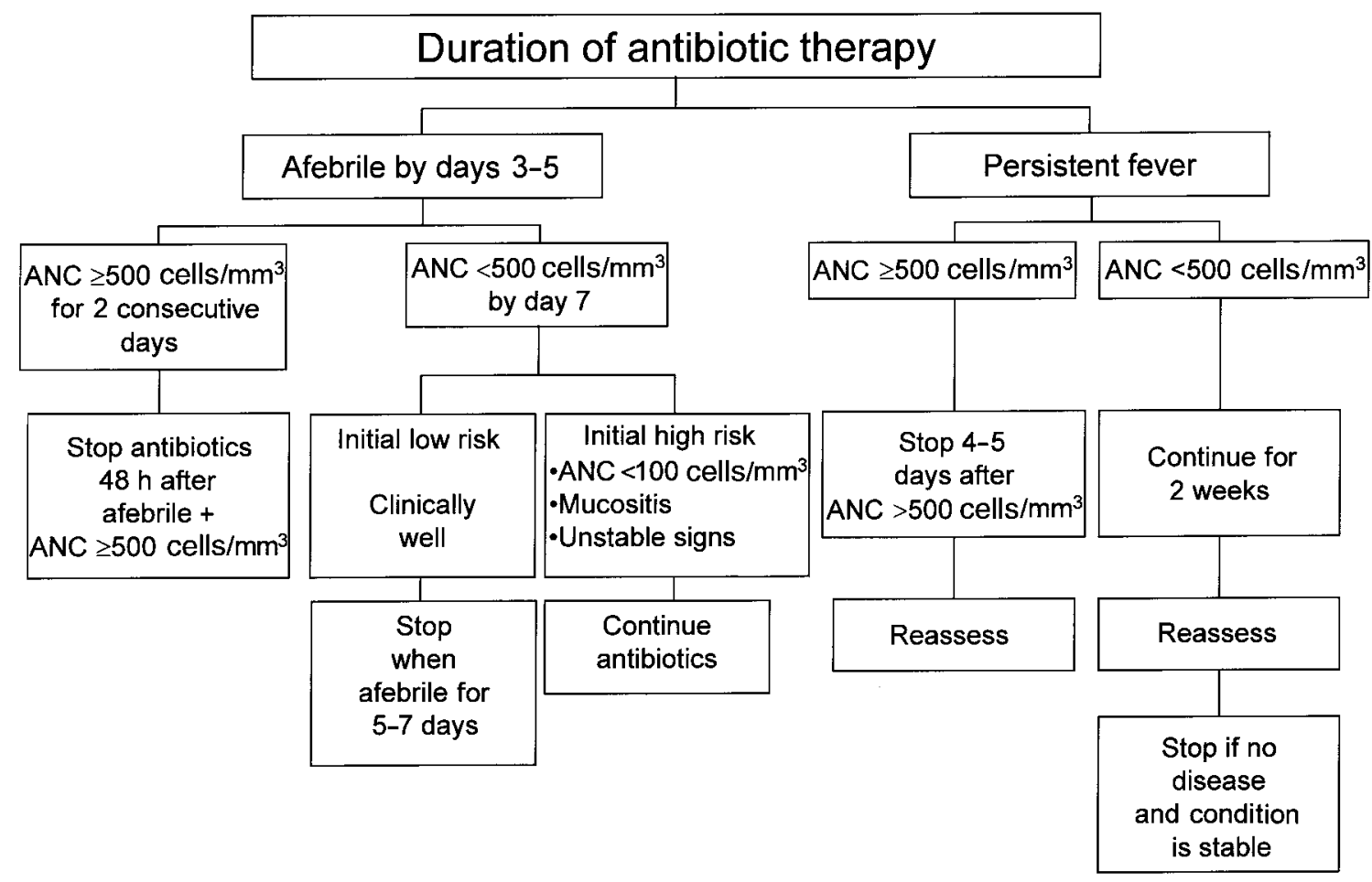

Figure 4. Suggested scheme for estimating the duration of antibiotic administration under various conditions. See tables 3 and 4 for rating system for patients at low risk. ANC, absolute neutrophil count.

other molds. Amphotericin B still provides a broader spectrum of activity than does fluconazole, although it does not appear to prevent subsequent aspergillosis $[123,124]$. A recent systematic meta-analytical review of lipid-based formulations of amphotericin B and the azoles against amphotericin B deoxycholate in febrile neutropenic patients showed no consistent treatment advantages associated with either formulation [125]. In a recent controlled study of 384 neutropenic patients with cancer, itraconazole and amphotericin B were equivalent in efficacy as empirical antifungal therapy, although itraconazole was associated with less toxicity [126].

Caspofungin, an echinocandin, has recently been approved by the FDA for the treatment of invasive aspergillosis refractory to amphotericin B and itraconazole. Data are not adequate for recommendations regarding its use for treatment of febrile neutropenic patients.

Recommendations if fever persists for $>3$ days. Figure 3 summarizes recommendations for patients with fever that persists for $>3$ days. Begin diagnostic reassessment after 3 days of treatment. By day 5 , if fever persists and reassessment is unrevealing, there are 3 options: (1) continue administration of the same antibiotic(s) if the patient's condition is clinically stable, (2) change antibiotics if there is evidence of progressive disease or drug toxicity, or (3) add an antifungal agent if the patient is expected to have neutropenia for longer than 5-7 more days (B-II).

\section{DURATION OF ANTIMICROBIAL THERAPY}

The single most important determinant of successful discontinuation of antibiotics is the neutrophil count. If no infection is identified after 3 days of treatment, if the neutrophil count is $\geqslant 500$ cells $/ \mathrm{mm}^{3}$ for 2 consecutive days, and if the patient is afebrile for $\geqslant 48 \mathrm{~h}$, antibiotic therapy may be stopped at that time (C-III) . If the patient becomes afebrile but remains neutropenic, the proper antibiotic course is less well defined. Some specialists recommend continuation of antibiotics, given intravenously or orally, until neutropenia is resolved (B-II) [108, $127,128]$. This approach may increase the risk for drug toxicity and superinfection with fungi or drug-resistant bacteria [129]. It is reasonable for neutropenic patients who appear healthy clinically, who were in a low risk category at onset of treatment, who have no discernible infectious lesions, and who have no radiographic or laboratory evidence of infection, to have their use of systemic antibiotics stopped after 5-7 afebrile days, or sooner, with evidence of hematologic recovery [44, 113, 127, $130,131]$. If use of antibiotics is stopped while the patient has neutropenia, the patient must be monitored closely and intravenous antibiotics restarted immediately on the recurrence of fever or other evidence of bacterial infection (figure 4) [132]. One should consider continuous administration of antibiotics throughout the neutropenic period in patients with profound neutropenia $\left(<100\right.$ cells $\left./ \mathrm{mm}^{3}\right)$, mucous membrane lesions of 
the mouth or gastrointestinal tract, unstable vital signs, or other identified risk factors (C-III). In patients with prolonged neutropenia in whom hematologic recovery cannot be anticipated, one can consider stopping antibiotic therapy after 2 weeks, if no site of infection has been identified and the patient can be observed carefully (C-III). Some experts suggest a change from the therapeutic regimen to one of the prophylactic schemes described below in the Antibiotic Prophylaxis for Afebrile Neutropenic Patients section [128, 129, 133].

The duration of amphotericin B therapy varies. If a systemic fungal infection has been identified, the course of antifungal therapy will be determined by the causative agent and the extent of the disease. However, if no fungal infection is found, it is not clear how long amphotericin B or other antifungal drugs should be administered. Experience is limited predominantly to amphotericin B. When neutropenia has resolved, the patient is clinically well, and CT of the abdomen and chest reveals no suspicious lesions, use of amphotericin B may be discontinued $[134,135]$. For clinically well patients with prolonged neutropenia, it is suggested that, after 2 weeks of receipt of daily doses of amphotericin B, if no discernible lesions can be found by clinical evaluation, chest radiography (or CT of the chest), and CT of abdominal organs [136, 137], use of the drug can be stopped. In the patient who appears ill or is at high risk, one should consider continuation of therapy with antibiotics and amphotericin B throughout the neutropenic episode, assuming that hematologic recovery can be anticipated.

Another approach to clinically well patients with persistent fever, which is preferred by other experts, is to terminate initial antibiotic therapy after $\sim 4$ days if no evidence of infection is found and there is no response to therapy (C-III). Under these conditions, which include very close, continuous monitoring of patients, subsequently demonstrated infections may occur, but most infections can be adequately treated [132]. Empirical amphotericin B administration should be considered for these patients, despite discontinuation of antibiotic therapy, if fever persists for 5-7 days after the start of initial therapy.

For patients who remain febrile after recovery of the neutrophil count to $\geqslant 500$ cells $/ \mathrm{mm}^{3}$ and despite receipt of broadspectrum antibacterial therapy, reassessment for undiagnosed infection should be directed at fungal (especially chronic systemic candidiasis, aspergillosis, histoplasmosis, and trichosporonosis), mycobacterial, or viral infections [138]. Antibiotic therapy can generally be stopped despite persistent fever 4-5 days after the neutrophil count reaches $\geqslant 500$ cells $/ \mathrm{mm}^{3}$ if no infectious lesions are identified. Ultrasonography (or, preferably, CT or MRI) of the abdomen may be useful for the detection of systemic fungal infections. Splenic, hepatic, and/or renal lesions may become apparent or enlarged as the neutrophil count increases.
Recommendations for duration of therapy. Recommendations for duration of therapy are summarized in figure 4 .

\section{THE USE OF ANTIVIRAL DRUGS}

There is usually no indication for the empirical use of antiviral drugs in the treatment of febrile neutropenic patients without evidence of viral disease. However, if skin or mucous membrane lesions due to herpes simplex or varicella-zoster viruses are present, even if they are not the cause of fever, treatment with acyclovir is indicated. The intent is to enhance the healing of these lesions, which provide portals of entry for bacteria and fungi during the neutropenic period. In certain patients with hematologic malignancies, fever, and neutropenia, the administration of acyclovir for the treatment or suppression of herpes simplex virus infection has been associated with a more favorable febrile response than that in untreated patients [139]. Newer agents, such as valacyclovir and famciclovir, are better absorbed after oral administration than is acyclovir and have longer dosing intervals, and they might be preferred to oral acyclovir. Systemic infections and disease due to cytomegalovirus are uncommon causes of fever in neutropenic patients, with the exception of those who have undergone bone marrow transplantation. Cytomegalovirus infection is treated with ganciclovir or foscarnet. The newer drugs of cidofovir, valganciclovir, and fomivirsen are effective for the treatment cytomegalovirus retinitis in patients with AIDS, but study among neutropenic patients has been inadequate.

If certain viral respiratory tract infections are identified soon after onset in the febrile neutropenic patient, use of suitable antiviral agents is usually warranted (e.g., ribavirin, for respiratory syncytial virus infection, and zanamivir, oseltamivir, rimantadine, or amantadine, for influenza virus infections).

Recommendations for use of antiviral drugs. Antiviral drugs are indicated only if there is clinical or laboratory evidence of viral disease.

\section{GRANULOCYTE TRANSFUSIONS}

The routine use of granulocyte transfusions is not usually advocated. However, for certain patients with profound neutropenia in whom the microbiologically documented causative bacteria cannot be controlled with optimal antibiotic therapy or by administration of a granulocyte colony-stimulating factor (G-CSF), and in cases of severe uncontrollable fungal infections, some investigators believe that granulocyte transfusions may be useful [140-142]. Transfusion of high numbers of granulocytes obtained after administration of G-CSF, with or without dexamethasone, to the donor is done by some clinicians, but, at this time, there is no convincing evidence of its efficacy. Significant toxicities in recipients include transmission of cy- 
tomegalovirus, alloimmunization associated with fever, graftversus-host reactions if granulocytes are not irradiated, progressive platelet refractoriness, and, possibly, respiratory insufficiency associated with concomitant administration of amphotericin B. The conclusion that granulocyte transfusion therapy is currently an experimental clinical practice seems warranted [143].

Recommendations. There are no specific indications for standard use of granulocyte transfusions (C-II).

\section{USE OF COLONY-STIMULATING FACTORS IN TREATMENT}

Hematopoietic growth factors have been studied as adjunctive therapy to antimicrobial therapy for febrile neutropenic patients in several randomized, controlled trials [144-155]. These studies show that G-CSF (filgrastim) or granulocyte-macrophage colony-stimulating factor (sargramostim) used as part of the treatment of febrile neutropenic patients can consistently shorten the duration of neutropenia, but these agents have not consistently and significantly reduced other measures of febrile morbidity, including duration of fever, use of anti-infectives, or costs of management of the febrile neutropenic episode. No study has demonstrated a decrease in infection-related mortality rates. The 2001 update of the American Society of Clinical Oncology guidelines recommends against the routine use of hematopoietic growth factor in uncomplicated cases of fever and neutropenia [156]. The IDSA panel agrees with these guidelines.

Under certain conditions in which worsening of the course is predicted and there is an expected long delay in recovery of the marrow, use of these agents may be indicated. Such conditions include pneumonia, hypotensive episodes, severe cellulitis or sinusitis, systemic fungal infections, and multiorgan dysfunction secondary to sepsis. Therapy with colony-stimulating factors could also be considered for patients who remain severely neutropenic and have documented infections that do not respond to appropriate antimicrobial therapy.

Recommendations for the use of colony-stimulating factors. Colony-stimulating factors are not recommended for routine use to treat febrile or afebrile neutropenic patients. The IDSA panel supports and endorses the American Society of Clinical Oncology guidelines (D-II) [156].

\section{ANTIBIOTIC PROPHYLAXIS FOR AFEBRILE NEUTROPENIC PATIENTS}

Since the 1980s, several studies have shown that the frequency of febrile episodes and infectious diseases can be reduced with the administration of antibiotics during the early afebrile period of neutropenia [1, 157-159]. Whatever benefit may come from the administration of necessarily broad-spectrum antibiotics is countered by deleterious effects from toxicity, emergence of antibiotic-resistant bacteria, and fungal overgrowth. Of special concern is the increasing prevalence of antibiotic-resistant bacteria.

Afebrile patients who are expected to be profoundly neutropenic $\left(<100\right.$ cells $\left./ \mathrm{mm}^{3}\right)$ are at greater risk for developing resistant infections than are those with counts of 500 cells $/ \mathrm{mm}^{3}$. Additional significant risk factors include lesions that break the mucous membranes and skin, use of indwelling catheters, use of instruments (e.g., endoscopy), severe periodontal disease, history of dental procedures, postobstructive pneumonia, status of malignancy or organ engraftment, and compromise of other immune responses. Personal factors, such as willingness to comply with prescribed prophylaxis, personal hygiene habits, and environmental (i.e., hospital or home) circumstances, must also be considered.

Combinations of nonabsorbable drugs, such as aminoglycosides, polymyxins, and vancomycin, have been used for infection prophylaxis in the past. Prospective, randomized trials have consistently shown that orally absorbable agents, such as trimethoprim-sulfamethoxazole (TMP-SMZ) and quinolones, are more effective and better tolerated for this purpose. In addition, the increasing frequency of antibiotic resistance strongly recommends against the use of prophylactic vancomycin.

Two types of oral absorbable antibiotics may be considered for chemoprophylaxis. These are TMP-SMZ and the quinolones.

TMP-SMZ. Studies of prophylaxis with TMP-SMZ were reviewed in the 1997 report of the IDSA Fever and Neutropenia Panel [1]. In most of these studies, the infection rates for TMPSMZ-treated patients were significantly lower than were those for placebo-treated control subjects, especially among patients who had neutropenia for $>2$ weeks after reinduction of cytotoxic therapy for leukemia. Adverse effects were few and insignificant, but bacterial resistance has been noted. TMP-SMZ has proven highly effective in the prevention of Pneumocystis carinii pneumonia in neutropenic and nonneutropenic patients [160].

Experts differ on recommendations regarding the routine use of TMP-SMZ during periods of neutropenia. These differences stem in great part from the overall lack of impact of prophylaxis on patient mortality. In some studies, periods of granulocytopenia were prolonged and the rate of fungal colonization was increased among patients receiving the antibiotics [160]. In patients at high risk for $P$. carinii pneumonitis (e.g., those with leukemia, certain solid tumors, histiocytosis, or AIDS), TMPSMZ is indicated to prevent the pneumonitis and will secondarily afford prophylaxis against some bacterial infections, whether or not neutropenia occurs. Disadvantages of this regimen include adverse reactions caused by sulfonamide drugs, myelosuppression (in some cases), development of drug-resistant bacteria, and oral candidiasis. Furthermore, the spectrum of TMP-SMZ does not include $P$. aeruginosa. 
Quinolones. The oral quinolones have been used extensively for prophylaxis in neutropenic patients [1, 157-159, 161-163], although they are ineffective against $P$. carinii. The results of comparative studies of ofloxacin or ciprofloxacin versus TMP-SMZ suggest that the effectiveness of the quinolones is equal or superior to that of TMP-SMZ for the prevention of febrile episodes of infectious origin [1]. Unfortunately, most of the studies have involved a number of patients that is inadequate for sound statistical analysis. However, 2 studies of reasonable size are of interest. The study by Kern and Kurrle [162] of 128 neutropenic patients who were randomized to receive either ofloxacin or TMP-SMZ showed that gram-negative bacillary infections occurred significantly less frequently in the ofloxacin group, but no difference in the frequency of gram-positive bacterial and fungal infections was noted. Bacteremia due to methicillin-resistant staphylococci may occur more frequently in neutropenic patients who receive quinolone prophylaxis than it does in those who have not previously received quinolone antibiotics [164]. Thus, a disadvantage of prophylaxis with quinolones is the inadequate coverage for gram-positive bacterial infections. In another study, the addition of penicillin significantly reduced the number of episodes of bacteremia, primarily via a reduction in the frequency of streptococcal bacteremia in the penicillin group [165]. The addition of rifampin to a quinolone resulted in more effective prophylaxis in 2 studies [159, 163] but not in another [157]. Three reports in which meta-analysis had been applied to estimate efficacy found that quinolone-based prophylaxis substantially reduced the incidence of fever and microbiologically documented infections in neutropenic patients [158, 166, 167]. The emergence of quinolone-resistant gram-negative bacilli has been demonstrated in patients given quinolone prophylaxis [168-171]. The quinolone drugs have not been approved by the FDA for infants and children. These antibiotics should not be used as prophylaxis routinely in medical centers in which resistance has already been observed or, if parenteral quinolones are part of empirical therapy, for febrile episodes in neutropenic patients. New fluoroquinolones with improved activity against gram-positive bacteria have become available, but clinical experience with their use to treat neutropenic patients is limited. The impact of the recent introduction of ciprofloxacin for anthrax prophylaxis on the development of resistance in other bacteria is unknown.

Vancomycin. Intravenous vancomycin has been used as prophylaxis for catheter-related or quinolone-related grampositive infections. Although this approach may be effective, it must be strongly discouraged because of the potential for emergence of vancomycin-resistant organisms. Furthermore, the newer drugs, linezolid and quinupristin-dalfopristin, should not be used for this purpose. Linezolid-related myelosuppression may occur.
Antifungal drugs. The frequency of fungal infections has increased substantially in recent years. Because these infections are often difficult to diagnose and treat successfully, antifungal prophylaxis may be appropriate in institutions in which the infections are encountered frequently. Fluconazole has been shown to reduce the frequency of both superficial and systemic infections in patients who undergo bone marrow transplantation [172-174]. Fluconazole's efficacy is limited by its lack of activity against C. krusei, some strains of C. glabrata, and molds. Increased frequency of colonization with C. krusei and C. glabrata has been reported in a few institutions in which fluconazole has been used [175]. A recent randomized, doubleblind trial of 274 adult neutropenic patients with cancer showed that fluconazole prophylaxis reduced the incidence of both superficial and invasive fungal infections and fungal-related deaths [176]. We agree with the 2000 guidelines from the CDC, IDSA, and American Society of Blood and Bone Marrow Transplantation [177], which recommends administration of fluconazole at a dosage of $400 \mathrm{mg} /$ day from the day of hematopoietic stem cell transplantation until engraftment for the prevention of candidiasis.

Two large randomized, double-blind trials have shown that itraconazole prophylaxis significantly reduced the frequency of systemic fungal infection due to Candida species, and 1 trial showed a decrease in the mortality rate associated with candidiasis $[178,179]$. A systematic meta-analytical review of the efficacy of antifungal prophylaxis among $>7000$ randomized subjects observed reductions in the use of empirical antifungal therapy, superficial fungal infection, invasive fungal infection, and the fungal infection-related mortality rate [180].

Recommendations for antimicrobial prophylaxis. TMPSMZ therapy is recommended for all patients at risk for $P$. carinii pneumonitis, regardless of whether they have neutropenia (A-I). However, there is no consensus to recommend TMP-SMZ or quinolones for routine use for all afebrile neutropenic patients. This lack of consensus is based, in great part, on the current concern about the emergence of antibioticresistant bacteria that has resulted from the overuse of antibiotics. In some special cases, for patients with profound and prolonged neutropenia, a quinolone plus penicillin or TMPSMZ may be considered for critical periods of time, if the potential for resistant organisms is appreciated and outweighed.

Routine use of fluconazole or itraconazole for all cases of neutropenia is not recommended (D-II). However, in certain circumstances in which the frequency of systemic infection due to Candida albicans is high and the frequency of systemic infection due to other Candida species and Aspergillus species is low, some physicians may elect to administer antifungal prophylaxis.

Our recommendations for routine prophylaxis are, in a sense, paradoxical. Data supporting the efficacy of prophylaxis with TMP-SMZ, quinolones, fluconazole, and itraconazole in re- 
ducing the number of infectious episodes during the neutropenic period are adequate and would warrant a rating of A-I from the standpoint of efficacy alone. However, concern about the problem of emerging drug-resistant bacteria and fungi due to extensive antibiotic use, plus the fact that such prophylaxis has not been shown to consistently reduce mortality rates, leads to the recommendation that routine prophylaxis with these drugs in neutropenic patients be avoided, with the exception of use of TMP-SMZ for patients at risk for $P$. carinii pneumonitis. An axiom for prophylaxis is that the antibiotic used should be administered for as short a period as possible and to as few patients as possible.

\section{ECONOMIC ISSUES}

Several approaches to reducing the cost of treating neutropenic patients with unexplained fever have been explored [131, 132, 181-184]. Opportunities to reduce costs have proliferated because of an expanding armamentarium of oral and intravenous antimicrobials, the emergence of hematopoietic colony-stimulating factors, the advent of home antibiotic therapy services, and data suggesting that empirical therapy can be discontinued early in certain subsets of low-risk patients [113-115]. When economic studies are conducted, it is essential that the welfare of patients be paramount. It is not sufficient to simply demonstrate statistically significant cost savings unless the impact on morbidity and mortality is also considered.

Outpatient treatment of low-risk episodes of fever and neutropenia is substantially less costly than inpatient care and is preferred by most patients and families [185-187]. The panel has attempted to encourage outpatient management when it is safe and feasible. An assumption that hospitalization of a patient is the safest course to take is not necessarily correct, in light of the Institute of Medicine's recent report that $>90,000$ preventable deaths occur in hospitals in the United States each year.

The dosage of a drug should be considered with regard to cost. Without question, the most effective dosage is basic for this decision. However, there is no need to exceed the optimal dosage. For example, the recommended dosage of ceftazidime is $2.0 \mathrm{~g}$ given every $8 \mathrm{~h}$ for treatment of patients with severe, life-threatening infections. However, in some studies, the lower dosage of $1.0 \mathrm{~g}$ given every $8 \mathrm{~h}$ has been used successfully to treat patients with solid tumors who have expected short periods of neutropenia [188]. Confirmation of such dosage schemes is needed before a practice standard is established.

Duration of antibiotic treatment beyond the reasonable periods mentioned here will obviously add to the cost of treatment and, at this point, would not seem warranted, except in special cases. The step-down from inpatient intravenous antibiotics to outpatient oral antibiotics is usually cost-efficient.
The expensive colony-stimulating factors are frequently used routinely, when they should be used according to well-thoughtout guidelines, such as those of the American Society of Clinical Oncology [156]. Under some circumstances, such as high-dose chemotherapy with either bone marrow or peripheral blood stem cell support, colony-stimulating factors may be both clinically and economically effective [189].

Liposomal and lipid-complex amphotericin B cost 10-60 times more than does amphotericin B deoxycholate and should be used only for the FDA-approved indications: for cases of aspergillosis that do not respond to the conventional amphotericin B preparation and for patients who cannot tolerate the conventional drug or who have or are at high risk for renal insufficiency. Avoidance of the indiscriminate use of antifungal and antiviral drugs during the febrile neutropenic episode requires adherence to the policy of use only when adequate scientific data support the indication.

A simplified approach to performing marginal cost-effectiveness analyses is detailed in a report from the Centers for Disease Control and Prevention [190] and requires a description of the program and of the health outcomes averted and the timing of these events; the rates of health outcomes and the preventable fraction of the health outcomes averted; the costs per unit of the intervention and the costs of the health outcomes prevented; and the side effects incurred. Another guide to decision analysis and economic evaluation is provided by Haddix et al. [191]. Because costs differ from location to location, the cost-effectiveness of an intervention in the management of fever and neutropenia must be determined at the physicians' respective hospitals.

\section{Acknowledgments}

We acknowledge with appreciation the following expert consultants who made valuable suggestions for the guidelines: Alain Cometta (Centre Hospitalier Universitaire Vaudois, Lausanne, Switzerland), Gerald R. Donowitz (University of Virginia, Charlottesville), Claudio Viscoli (University of Genova, Genova, Italy), and James C. Wade (Fred Hutchinson Cancer Research Center, Seattle).

\section{References}

1. Hughes WT, Armstrong D, Bodey GP, et al. 1997 guidelines for the use of antimicrobial agents in neutropenic patients with unexplained fever. Infectious Diseases Society of America. Clin Infect Dis 1997; 25:551-73.

2. Bodey GP, Buckley M, Sathe YS, Freireich EJ. Quantitative relationships between circulating leukocytes and infection in patients with acute leukemia. Ann Intern Med 1966; 64:328-40.

3. Schimpff SC. Empiric antibiotic therapy for granulocytopenic cancer patients. Am J Med 1986; 80:13-20.

4. Lucas KG, Brown AE, Armstrong D, et. al. The identification of febrile, 
neutropenic children with neoplastic disease at low risk for bacteremia and complications of sepsis. Cancer 1996; 77:791-8.

5. Dale DC, Guerry D 4th, Wewerka JR, Chusid MJ. Chronic neutropenia. Medicine (Baltimore) 1979; 58:128-44.

6. Sickles EA, Greene WH, Wiernik PH. Clinical presentation of infection in granulocytopenic patients. Arch Intern Med 1975; 135:715-9.

7. Mermel LA, Farr BM, Sheretz RJ, et al. Guidelines for the management of intravascular catheter-related infection. Clin Infect Dis 2001;32: $1249-72$.

8. Weinstein MP. Current blood culture methods and systems: clinical concepts, technology, and interpretation of results. Clin Infect Dis 1996; $23: 40-60$.

9. Adamkiewicz TV, Lorenzana A, Doyle J, et al. Peripheral vs. central blood cultures in patients admitted to a pediatric oncology ward. Pediatr Infect Dis J 1999; 18:556-8.

10. Whimbey E, Wong B, Kiehn TE, Armstrong D. Clinical correlations of serial quantitative blood cultures determined by lysis-centrifugation in patients with persistent septicemia. J Clin Microbiol 1984; 19: 766-71.

11. Rolston RVI, Balakrishnan M, Elting LS, et al. Is quantitative variation in cancer patients with bacteremic infections linked to severity of infection? [abstract 729]. In: Program and abstracts of the 40th Interscience Conference for Antimicrobial Agents and Chemotherapy (Toronto). Washington, DC: American Society for Microbiology, 2000.

12. McDonald LC, Weinstein MP, Mirrett S, et al. Controlled comparison of BacT/ALERT FAN aerobic medium and VATEC fungal blood culture medium for detection of fungemia. J Clin Microbiol 2001;39: $622-4$.

13. Weinstein MP, Mirrett S, Wilson ML, et al. Controlled evaluation of 5 versus 10 milliliters of blood cultured in aerobic BacT/Alert blood culture bottles. J Clin Microbiol 1994; 32:2103-6.

14. Flynn PM, Van Hooser B, Gigliotti F. Atypical mycobacterial infections of Hickman catheter exit sites. Pediatr Infect Dis J 1988; 7:510-3.

15. Bodey GP, Fainstein V. Systemic candidiasis. In: Bodley GP, Fainstein V, eds. Candidiasis. New York: Raven Press, 1985:135-68.

16. Guerrant RL, Van Gilder T, Steiner TS, et al. Practice guidelines for the management of infectious diarrhea. Clin Infect Dis 2001;32: $331-50$.

17. Heussel CP, Kauczor HU, Heussel GE, et al. Pneumonia in febrile neutropenic patients and in bone marrow and blood stem cell transplant recipients: use of high resolution computed tomography. J Clin Oncol 1999; 17:796-805.

18. Allen U, Smith CR, Prober CG. The value of skin biopsies in febrile, neutropenic, immunocompromised children. Am J Dis Child 1986; 140:459-61.

19. Lehrnbecher T, Venzon D, deHaas M, et al. Assessment of measuring circulating levels of interleukin-6, interleukin-8, C-reactive protein, soluble $\mathrm{Fc} \gamma$ receptor type III, and mannose-binding protein in febrile children with cancer and neutropenia. Clin Infect Dis 1999; 29:414-9.

20. Engel A, Steinbach G, Kern P, et al. Diagnostic value of procalcitonin serum levels in neutropenic patients with fever: comparison with interleukin-8. Scand J Infect Dis 1999;31:185-9.

21. Kern WV, Heiss M, Steinbach G, et al. Prediction of gram-negative bacteremia in patients with cancer and febrile neutropenia by means of interleukin-8 levels in serum: targeting empirical monotherapy versus combination therapy. Clin Infect Dis 2001;32:832-5.

22. Engel A, Mack E, Kern P, et al. An analysis of interleukin-8, interleukin-6, and C-reactive protein serum concentrations to predict fever, gram-negative bacteremia, and complicated infection in neutropenic cancer patients. Infection 1998;26:213-21.

23. Bochud PY, Calandra T, Francioli P. Bacteremia due to viridans streptococci in neutropenic patients: a review. Am J Med 1994; 97:256-64.

24. Pizzo PA, Ladisch S, Robichaud K. Treatment of gram-positive septicemia in cancer patients. Cancer 1980; 45:206-7.

25. Rolston KVI, Tarrand JJ. Pseudomonas aeruginosa-still a frequent pathogen in patients with cancer: 11-year experience at a comprehensive cancer center. Clin Infect Dis 1999;29:463-4.

26. Escande MC, Hebrect R. Prospective study of bacteremia in cancer patients. Support Care Cancer 1998; 6:273-80.

27. Collioud D, van der Auwera P, Viot M, et al. Prospective multicentric study of the etiology of 1051 bacteremic episodes in 782 cancer patients. Support Care Cancer 1993; 1:34-6.

28. Al-Bahar S, Pandita R, Dhabhar BN, et al. Febrile neutropenia in cancer patients in Kuwait: microbial spectrum and outcome. Support Care Cancer 1994; 2:400-2.

29. Spanik S, Stopkova K, Grausova S, et al. Postoperative bacteremia in cancer patients with solid tumors undergoing surgery: risk factors, etiology and outcome in 276 patients. Support Care Cancer 1997; 5: 336-8.

30. European Organization for Research and Treatment of Cancer (EORTC) International Antimicrobial Therapy Cooperative Group, National Cancer Institute of Canada-Clinical Trials Group. Vancomycin added to empirical combination antibiotic therapy for fever in granulocytopenic cancer patients. J Infect Dis 1991; 163:951-8.

31. Douard MC, Arlet G, Longuet P, et al. Diagnosis of venous access port-related infections. Clin Infect Dis 1999;29:1197-202.

32. Benezra D, Kiehn TE, Gold JW, et al. Prospective study of infections in indwelling central venous catheters using quantitative blood cultures. Am J Med 1988; 85:495-8.

33. Raad II, Vartivarian S, Khan A, et al. Catheter-related infections caused by the Mycobacterium fortuitum complex: 15 cases and review. Rev Infect Dis 1991; 13:1120-5.

34. Viscoli C, Bruzzi P, Glauser M. An approach to the design and implementation of clinical trails of empirical antibiotic therapy in febrile and neutropenic cancer patients. Eur J Cancer 1995;31A:2013-22.

35. Hughes WT, Pizzo PA, Wade JC, et al. Evaluation of new anti-infective drugs for the treatment of febrile episodes in neutropenic patients. Clin Infect Dis 1992; 15(Suppl 1):S206-15.

36. Johnson MP, Ramphal R. $\beta$-lactam-resistant Enterobacter bacteremia in febrile neutropenic patients receiving monotherapy. J Infect Dis $1990 ; 162: 981-3$.

37. Anaissie EJ, Fainstein V, Bodey GP, et al. Randomized trial of betalactam regimens in febrile neutropenic cancer patients. Am J Med 1988; 84:581-9.

38. Bodey GP, Fainstein V, Elting LS, et al. Beta-lactam regimens for the febrile neutropenic patient. Cancer 1990;65:9-16.

39. Malik IA, Khan WA, Karim M, et al. Feasibility of outpatient management of fever in cancer patients with low-risk neutropenia: results of a prospective randomized trial. Am J Med 1995; 98:224-31.

40. Mullen CA, Petropoulos D, Roberst WM, et al. Outpatient treatment of fever and neutropenia for low risk pediatric cancer patients. Cancer 1999; 86:126-34.

41. Garcia-Carbonero R, Cortes-Funes H. Outpatient therapy with oral ofloxacin for patients with low risk neutropenia and fever: a prospective, randomized clinical trial. Cancer 1999; 85:213-9.

42. Rolston KVI. New trends in patient management: risk-based therapy for febrile patients with neutropenia. Clin Infect Dis 1999; 29:515-21.

43. Freifeld A, Marchigiani D, Walsh T, et al. A double-blind comparison of empirical oral and intravenous antibiotic therapy for low-risk febrile patients with neutropenia during cancer chemotherapy. N Engl J Med 1999; 341:305-11.

44. Aquino VM, Tkaczewski I, Buchanan GR. Early discharge of low-risk febrile neutropenic children and adolescents with cancer. Clin Infect Dis $1997 ; 25: 74-8$.

45. Kern WV, Cometta A, DeBock R, et al. Oral versus intravenous empirical antimicrobial therapy for fever in patients with granulocytopenia who are receiving cancer chemotherapy. N Engl J Med 1999; 341:312-8.

46. Klaasseen RJ, Goodman R, Pham BA, Doyle JJ. "Low-risk” prediction rule for pediatric oncology patients presenting with fever and neutropenia. J Clin Oncol 2000; 18:1012-9.

47. Rackoff WR, Gonin R, Robinson C, et al. Predicting the risk of bac- 
teremia in children with fever and neutropenia. J Clin Oncol 1996; 14:919-24.

48. Jones GR, Konsler GK, Dunaway RP, et al. Risk factors for recurrent fever after the discontinuation of empiric antibiotic therapy for fever and neutropenia in pediatric patients with a malignancy or hematologic condition. J Pediatr 1994; 124:703-8.

49. Elting LS, Rubenstein EB, Rolston KVI, et al. Outcome of bacteremia in patients with cancer and neutropenia: observations from two decades of epidemiological and clinical trials. Clin Infect Dis 1997;25: $247-59$.

50. Klastersky J, Paesmans M, Rubenstein EB, et al. The multinational association for supportive care in cancer risk index: a multinational scoring system for identify low-risk febrile neutropenic cancer patients. J Clin Oncol 2000; 18:3038-51.

51. Talcott JA, Siegel RD, Finberg R, et al. Risk assessment in cancer patients with fever and neutropenia: a prospective, two-center validation of a prediction rule. J Clin Oncol 1992; 10:316-22.

52. Shenep JL, Flynn PM, Baker DK, et al. Oral cefixime is similar to continued intravenous antibiotics in the empirical treatment of febrile neutropenic children with cancer. Clin Infect Dis 2001;32:36-43.

53. Paganini HR, Sarkis CM, DeMartino MG, et al. Oral administration of cefixime to lower risk febrile neutropenic children with cancer. Cancer 2000; 88:2848-52.

54. Rubinstein E, Lode H, Grassi C. Ceftazidime monotherapy vs. ceftriaxone/tobramycin for serious hospital-acquired gram-negative infections. Antibiotic Study Group. Clin Infect Dis 1995;20:1217-28.

55. Winston DJ, Ho WG, Bruckner DA, Champlin RE. Beta-lactam antibiotic therapy in febrile granulocytopenic patients: a randomized trial comparing cefoperazone plus piperacillin, ceftazidime plus piperacillin, and imipenem alone. Ann Intern Med 1991;115:849-59.

56. de Pauw BE, Deresinski SC, Feld R, et al. Ceftazidime compared with piperacillin and tobramycin for the empiric treatment of fever in neutropenic patients with cancer: a multicenter randomized trial. Ann Intern Med 1994; 120:834-44.

57. Pizzo PA, Hathorn JW, Hiemenz J, et al. A randomized trial comparing ceftazidime alone with combination antibiotic therapy in cancer patients with fever and neutropenia. N Engl J Med 1986;315:552-8.

58. Gardembas-Pain M, Desablens B, Sensebe L, et al. Home treatment of febrile neutropenia: an empirical oral antibiotic regimen. Ann Oncol 1991; 2:485-7.

59. Malik IA, Abbas A, Karim M. Randomized comparison of oral ofloxacin alone with combination of parenteral antibiotics in neutropenic febrile patients. Lancet 1992;339(8801):1092-6.

60. Wang FD, Liu CY, Hsu HC, et al. A comparative study of cefepime versus ceftazidime as empiric therapy of febrile episodes in neutropenic patients. Chemotherapy 1999; 45:370-9.

61. Biron P, Fuhrmann C, Cure H, et al. Cefepime versus imipenemcilastatin as empirical monotherapy in 400 febrile patients with short duration neutropenia: CEMIC. J Antimicrob Chemother 1998; 42 : 511-8.

62. Lindbald R, Rodjer S, Adriansson M, et al. Empiric monotherapy for febrile neutropenia - a randomized study comparing meropenem with ceftazidime. Scand J Infect Dis 1998;30:237-43.

63. Behre G, Link H, Maschmeyer G, et al. Meropenem monotherapy versus combination therapy with ceftazidime and amikacin for empirical treatment of febrile neutropenic patients. Ann Hematol 1998; 76:73-80.

64. Bohme A, Shah PM, Stille W, et al. Piperacillin/tazobactam versus cefepime as initial empirical antimicrobial therapy in febrile neutropenia patients: a prospective randomized pilot study. Eur J Med Res $1998 ; 3: 324-30$.

65. Del Favero A, Menichetti F, Martino P, et al. A multicenter, doubleblind, placebo-controlled trial comparing piperacillin-tazobactam with and without amikacin as empiric therapy for febrile neutropenia. Clin Infect Dis 2001;33:1295-301.

66. Engervall P, Kalin M, Dornbusch K, et al. Cefepime as empirical monotherapy in febrile patients with hematological malignancies and neutropenia: a randomized, single-center phase II trail. J Chemother 1999; 11:278-86.

67. Ozyilkan O, Yalcintas U, Baskan S. Imipenem-cilastatin versus sulbactam-cefoperazone plus amikacin in the initial treatment of febrile neutropenic cancer patients. Korean J Intern Med 1999; 14:15-9.

68. Ramphal R. Is monotherapy for febrile neutropenia still a viable alternative? Clin Infect Dis 1999;29:508-14.

69. Akova M, Akan H, Korten H, et al. Comparison of meropenem with amikacin plus ceftazidime in the empirical treatment of febrile neutropenia: a prospective randomized multicentre trial in patients without previous prophylactic antibiotics. Meropenem Study Group of Turkey. Int J Antimicrob Agents 1999; 13:15-9.

70. Yamamura D, Gacalp R, Carlisle P, et al. Open randomized study of cefepime versus piperacillin-gentamicin for treatment of febrile neutropenic cancer patients. Antimicrob Agents Chemother 1997; 41: $1704-8$.

71. Cometta A, Calandra T, Gaya H, et al. Monotherapy with meropenem versus combination therapy with ceftazidime plus amikacin as empiric therapy for fever in granulocytopenic patients with cancer. Antimicrob Agents Chemother 1996; 40:1108-15.

72. Raad II, Abi-Said D, Rolston KV, et al. How should imipenem-cilastatin be used in the treatment of fever and infection in neutropenic cancer patients? Cancer 1998; 82:2449-58.

73. Oblon D, Ramphal R. A randomized trial of cefepime vs. ceftazidime as initial therapy for patients with prolonged fever and neutropenia after intensive chemotherapy [abstract]. In: Proceedings of the annual meeting of the American Association for Cancer 1993; 34:1362A.

74. Feld R, DePauw B, Berman S, et al. Meropenem versus ceftazidime in the treatment of cancer patients with febrile neutropenia: a randomized, double-blind trial. J Clin Oncol 2000; 18:3690-8.

75. Owens RC, Owens CA, Holloway WJ. Reduction in vancomycin consumption in patients with fever and neutropenia [abstract 458]. Clin Infect Dis 2000; $31: 291$.

76. Vandercam B, Gerain J, Humblet Y, et al. Meropenem versus ceftazidime as empirical monotherapy for febrile neutropenic patients. Ann Hematol 2000; 79:152-7.

77. Johnson PRE, Liu Yin JA, Tooth JA. High dose intravenous ciprofloxacin in febrile neutropenic patients. J Antimicrob Chemother 1990;26:101-7.

78. Johnson PR, Liu Yin JA, Tooth JA. A randomized trial of high-dose ciprofloxacin versus azlocillin and netilmicin in the empirical therapy of febrile neutropenic patients. J Antimicrob Chemother 1992; 30: 203-14.

79. Winston DJ, Lazarus HM, Beveridge RA, et al. Randomized, doubleblind multicenter trail comparing clinafloxacin with imipenem as empirical monotherapy for febrile granulocytopenic patients. Clin Infect Dis 2001;32:381-90.

80. Malik IA, Abbas Z, Karim M. Randomized comparison of oral ofloxacin alone with combination of parenteral antibiotics in neutropenic febrile patients. Lancet 1992; 339:1092-6.

81. Meunier F, Zinner SH, Gaya H, et al. Prospective randomized evaluation of ciprofloxacin versus piperacillin plus amikacin for empiric antibiotic therapy of febrile granulocytopenic cancer patients with lymphomas and solid tumor. European Organization for Research on Treatment of Cancer International Antimicrobial Therapy Cooperative Group. Antimicrob Agents Chemother 1991;35:873-8.

82. Klastersky J, Vamecq G, Cappel R, Swings G, Vandenborre L. Effects of the combination of gentamicin and carbenicillin on the bactericidal activity of serum. J Infect Dis 1972; 125:183-6.

83. Sepkowitz KA, Brown AE, Armstrong D. Empirical therapy for febrile, neutropenic patients: persistence of susceptibility of gram-negative bacilli to aminoglycoside antibiotics. Clin Infect Dis 1994; 19:810-1.

84. Brown AE, Kiehn TE, Armstrong D. Bacterial resistance in the patient with neoplastic disease. Infect Dis Clin Pract 1995; 4:136-44.

85. International Antimicrobial Therapy Cooperative Group of the Eu- 
ropean Organization for Research and Treatment of Cancer. Efficacy and toxicity of single daily doses of amikacin and ceftriaxone versus multiple daily doses of amikacin and ceftazidime for infection in patients with cancer and granulocytopenia. Ann Intern Med 1993; 119:584-93.

86. Diekema DJ, Jones RN, Rolston KVI. Antimicrobial activity of gatifloxacin compared to seven other compounds tested against grampositive organisms isolated at 10 cancer-treatment centers. Diagn Microbiol Infect Dis 1999; 34:37-43.

87. Rolston KVI, LaBlanc BM, Balakrishnan M, et al. In-vitro activity of moxifloxacin against gram-negative isolates from cancer patients [abstract 2324]. In: Program and abstracts of the 40th Interscience Conference on Antimicrobial Agents and Chemotherapy (Toronto). Washington, DC: American Society for Microbiology, 2000.

88. Rolston KVI, LeBlanc H, Ho DH. In vitro activity of gatifloxacin against gram-negative isolates from cancer patients [abstract 359]. In: Program and abstracts of the 39th Interscience Conference on Antimicrobial Agents and Chemotherapy (San Francisco). Washington, DC: American Society for Microbiology, 1999.

89. Papadimitris C, Dimopoulos MA, Kostis E, et al. Outpatient treatment of neutropenic fever with oral antibiotics and granulocyte colonystimulating factor. Oncology 1999; 57:127-30.

90. Peacock JE, Wade JC, Lazarus HM, et al. Ciprofloxacin/piperacillin vs. tobramycin/piperacillin as empiric therapy for fever in neutropenic cancer patients, a randomized, double-blind trial [abstract 373]. In: Program and abstracts of the 37th Interscience Conference on Antimicrob Agents and Chemotherapy (Toronto). Washington, DC: American Society for Microbiology, 1997.

91. Chatzinikalaou I, Abi-Said D, Bodey GP, et al. Recent experience with Pseudomonas aeruginosa bacteremia in patients with cancer: retrospective analysis of 245 episodes. Arch Intern Med 2000; 160:501-9.

92. Centers for Disease Control and Prevention. Recommendations for preventing the spread of vancomycin resistance: recommendations of the Hospital Infection Control Practices Advisory Committee (HICPAC). MMWR Morb Mortal Wkly Rep 1995;44(RR-12):1-13.

93. Feld R. Vancomycin as part of initial empirical antibiotic therapy for febrile neutropenia in patients with cancer: pros and cons. Clin Infect Dis $1999 ; 29: 503-7$.

94. Shenep JL, Hughes WT, Roberson PK, et al. Vancomycin, ticarcillin, and amikacin compared with ticarcillin-clavulanate and amikacin in the empirical treatment of febrile, neutropenic children with cancer. N Engl J Med 1988; 319:1053-8.

95. Elting LS, Bodey GP, Keefe BH. Septicemia and shock syndrome due to viridans streptococci: a case-control study of predisposing factors. Clin Infect Dis 1992; 14:1201-7.

96. Traub WH, Spohr M, Bauer D. In vitro additive effect of imipenem combined with vancomycin against multiple-drug resistant, coagulase-negative staphylococci. Zentralbl Mikrobiol Hyg [A] 1986;262: 361-9.

97. Harvey WH, Harvey JH, Moskowitz MJ. Ciprofloxacin/vancomycin $(\mathrm{C} / \mathrm{V})$ as initial empiric therapy in febrile neutropenic leukemia/lymphoma patients (pts) with indwelling venous access devices: preliminary results of an effective regimen with reduced hospital stay [abstract A1639]. In: Proceedings of the annual meeting of the American Society of Clinical Oncology, 1994.13.

98. Kelsey SM, Shaw E, Newland AC. Aztreonam plus vancomycin versus gentamicin plus piperacillin as empirical therapy for the treatment of fever in neutropenic patients: a randomized controlled study. J Chemother 1992; 4:107-13.

99. Raad II, Whimbey EE, Rolston KVI, et al. A comparison of aztreonam plus vancomycin and imipenem plus vancomycin as initial therapy for febrile neutropenic cancer patients. Cancer 1996; 77:1386-94.

100. Ramphal R, Bolger M, Oblon DJ, et al. Vancomycin is not an essential component of the initial empiric treatment regimen for febrile neutropenic patients receiving ceftazidime: a randomized prospective study. Antimicrob Agents Chemother 1992;36:1062-7.
101. Cony-Makhoul P, Brossard G, Marit G, et al. A prospective study comparing vancomycin and teicoplanin as second-line empiric therapy for infection in neutropenic patients. Br J Haematol 1990; 76(Suppl 2):35-40.

102. Pico JL, Marie JP, Chiche D, et al. Should vancomycin be used empirically in febrile patients with prolonged and profound neutropenia? Results of a randomized trial. Eur J Med 1993;2:275-80.

103. Viscoli C, Moroni C, Boni L, et al. Ceftazidime plus amikacin versus ceftazidime plus vancomycin as empiric therapy in febrile neutropenic children with cancer. Rev Infect Dis 1991; 13:397-404.

104. Rikonen P. Imipenem compared with ceftazidime plus vancomycin as initial therapy for fever in neutropenic children with cancer. Pediatr Infect Dis J 1991; 10:918-23.

105. Chow AW, Jewesson PJ, Kureishi A, et al. Teicoplanin versus vancomycin in the empirical treatment of febrile neutropenic patients. Eur J Haematol Suppl 1993; 54:18-24.

106. Kureishi A, Jewesson PJ, Rubinger M, et al. Double-blind comparison of teicoplanin versus vancomycin in febrile neutropenic patients receiving concomitant tobramycin and piperacillin: effect on cyclosporin-associated nephrotoxicity. Antimicrob Agents Chemother 1991; 35:2246-52.

107. Rubinstein E, Cammarata SK, Oliphant TH, et al. Linezolid (PNU100766) versus vancomycin in the treatment of hospitalized patients with nosocomial pneumonia: a randomized, double-blind, multicenter study. Clin Infect Dis 2001;32:402-12.

108. Freifeld A, Pizzo P. Use of fluoroquinolones for empirical management of febrile neutropenia in pediatric cancer patients. Pediatr Infect Dis J 1997; 16:140-6.

109. Charnas R, Luthi AR, Ruch W. Once daily ceftriaxone plus amikacin vs. three times daily ceftazidime plus amikacin for treatment of febrile neutropenic children with cancer. Pediatr Infect Dis J 1997; 16:346-53.

110. Eggimann P, Glauser MP, Aoun M, Meunier F, Calandra T. Cefepime monotherapy for the empirical treatment of fever in granulocytopenic cancer patients. J Antimicrob Chemother 1993; 32(Suppl B):151-63.

111. Bow EJ, Loewen R, Vaughan D. Reduced requirement for antibiotic therapy targeting gram-negative organisms in febrile, neutropenic patients with cancer who are receiving antibacterial chemoprophylaxis with oral quinolones. Clin Infect Dis 1995;20:907-12.

112. Elting LS, Rubenstein EB, Rolston K, et al. Time to clinical response: an outcome of antibiotic therapy of febrile neutropenia with implications for quality and cost of care. J Clin Oncol 2000; 18:3699-706.

113. Mullen CA, Buchanan GR. Early hospital discharge of children with cancer treated for fever and neutropenia: identification and management of the low-risk patient. J Clin Oncol 1990; 8:1998-2004.

114. Jones GR, Konsler GK, Dunaway RP, et al. Risk factors for recurrent fever after the discontinuation of empiric antibiotic therapy for fever and neutropenia in pediatric patients with a malignancy or hematologic condition. J Pediatr 1994; 124:703-8.

115. Griffin TC, Buchanan GR. Hematologic predictors of bone marrow recovery in neutropenic patients hospitalized for fever: implications for discontinuation of antibiotics and early discharge from the hospital. J Pediatr 1992; 121:28-33.

116. Santolaya ME, Villarroel M, Avendano LF, et al. Discontinuation of antimicrobial therapy for febrile, neutropenic children with cancer: a prospective study. Clin Infect Dis 1997;25:92-7.

117. Woo PC, Wong SS, Lum PN, et al. Cell-wall deficient bacteria and culture-negative febrile episodes in bone marrow recipients. Lancet 2001; 357:675-9.

118. Pizzo PA, Robichaud KJ, Gill FA, Witebsky FG. Empiric antibiotic and antifungal therapy for cancer patients with prolonged fever and granulocytopenia. Am J Med 1982; 72:101-11.

119. EORTC International Antimicrobial Therapy Cooperative Project Group. Empiric antifungal therapy in febrile granulocytopenic patients. Am J Med 1989; 86:668-72.

120. Walsh TJ, Finberg RW, Arndt C, et al. Liposomal amphotericin B for empirical therapy in patients with persistent fever and neutropenia. 
National Institute of Allergy and Infectious Diseases Mycoses Study Group. N Engl J Med 1999; 340:764-71.

121. Wingard JR, White MH, Anaissie E, et al. A randomized, doubleblind comparative trial evaluating the safety of liposomal amphotericin B versus amphotericin lipid complex in the empirical treatment of febrile neutropenia. Clin Infect Dis 2000;31:1155-63.

122. Fleming RV, Kantarjian HM, Husni R, et al. Comparison of amphotericin B lipid complex (ABLC) vs. AmBisome in the treatment of suspected or documented fungal infections in patients with leukemia. Leuk Lymphoma 2001; 40:511-20.

123. Viscoli C, Castagnola E, VanLint MT, et al. Fluconazole versus amphotericin $\mathrm{B}$ as empirical antifungal therapy of unexplained fever in granulocytopenic cancer patients. Eur J Cancer 1996;32A:814-20.

124. Winston DJ, Hathorn JW, Schuster MG, et al. A multi-center randomized trial of fluconazole versus amphotericin B for empiric antifungal therapy of febrile neutropenic patients with cancer. Am J Med 2000; 108:282-9.

125. Bow EJ, Laverdiere M, Rotstein C. A systematic review of the efficacy of azoles and lipid-based formulations of amphotericin B as empirical antifungal therapy in persistently febrile neutropenic patients despite broad-spectrum antibacterial therapy [abstract]. In: Program and abstracts of the 40th Interscience Conference on Antimicrobial Agents and Chemotherapy (Toronto). Washington, DC: American Society for Microbiology, 2000.

126. Boogaerts M, Winston DJ, Bow EJ, et al. Intravenous and oral itraconazole versus intravenous amphotericin B deoxycholate as empirical antifungal therapy for persistent fever in neutropenic patients with cancer who are receiving broad-spectrum antibacterial therapy: a randomized, controlled trial. Ann Intern Med 2001; 135:412-22.

127. Pizzo PA, Robichaud KJ, Gill FA, et al. Duration of empiric antibiotic therapy in granulocytopenic patients with cancer. Am J Med 1979; 67:194-200.

128. Hughes WT, Patterson G. Post-sepsis prophylaxis in cancer patients. Cancer 1984; 53:137-41.

129. Crane L, Komskian S, Sauber A, et al. Antibiotic therapy in febrile neutropenic patients: what is the optimum duration of therapy? [abstract]. In: Program and abstracts of the 28th Interscience Conference on Antimicrobial Agents and Chemotherapy (Los Angeles). Washington, DC: American Society for Microbiology, 1988:114.

130. DeMarie S, Van den Broek PJ, Willemze R, et al. Strategy for antibiotic therapy in febrile neutropenic patients on selective antibiotic decontamination. Eur J Clin Microbiol Infect Dis 1993; 12:897-906.

131. Bash RO, Katz JA, Cash JV, et al. Safety and cost effectiveness of early hospital discharge of lower risk children with cancer admitted for fever and neutropenia. Cancer 1994; 74:189-96.

132. Joshi JH, Schimpff SC, Tenney JH, et al. Can antibacterial therapy be discontinued in persistently febrile granulocytopenic cancer patients? Am J Med 1984; 76:450-7.

133. Cornelissen JJ, Rozenberg-Arska M, Dekker AW. Discontinuation of intravenous antibiotic therapy during persistent neutropenia in patients receiving prophylaxis with oral ciprofloxacin. Clin Infect Dis 1995; $21: 1300-2$.

134. Santhosh-Kumar CR, Ajarim DSS, Harakati MS, et al. Ceftazidime and amikacin as empiric treatment of febrile episodes in neutropenic patients in Saudi Arabia. J Infect 1992; 25:11-9.

135. Heussel CP, Hauczor HU, Heussel G, et al. Early detection of pneumonia in febrile neutropenic patients: use of thin-section CT. AJR Am J Roentgenol 1997; 169:1347-53.

136. Bartley DL, Hughes WT, Parvey LS, et al. Computed tomography of hepatic and splenic fungal abscesses in leukemic children. Pediatr Infect Dis 1982; 1:317-21.

137. Flynn PM, Shenep JL, Crawford R, et al. Use of abdominal computed tomography for identifying disseminated fungal infection in pediatric cancer patients. Clin Infect Dis 1995; 20:964-70.

138. Talbot GH, Provencher M, Cassileth PA. Persistent fever after recovery from granulocytopenia in acute leukemia. Arch Intern Med 1988; 148:129-35.
139. Baglin TP, Gray JJ, Marcus RE, Wreghitt TG. Antibiotic resistant fever associated with herpes simplex virus infection in neutropenic patients with haematological malignancy. J Clin Pathol 1989;42:1255-8.

140. Bhatia S, McCullough J, Perry EH. Granulocyte transfusions: efficacy in treating fungal infections in neutropenic patients following bone marrow transplantation. Transfusion 1994; 34:226-32.

141. Catalano L, Fiontana R, Scarpato N, et al. Combined treatment with amphotericin B and granulocyte transfusion from G-CSF-stimulated donors in an aplastic patient with invasive aspergillosis undergoing bone marrow transplantation. Haematologica 1997; 82:71-2.

142. Dignani CM, Anaissie EJ, Hester JP, et al. Treatment of neutropeniarelated fungal infections with granulocyte colony-stimulating factor-elicited white blood cell transfusions: a pilot study. Leukemia 1997; 11:1621-30.

143. Hubel K, Dale DC, Engert A, Liles WC. Current status of granulocyte (neutrophil) transfusion therapy for infectious diseases. J Infect Dis 2001; 183:321-8.

144. Riikonen P, Saarinen UM, Makipernaa A. Recombinant human granulocyte-macrophage colony-stimulating factor in the treatment of febrile neutropenia: a double blind placebo-controlled study in children. Pediatr Infect Dis J 1994; 13:197-202.

145. Anaissie EJ, Vartivarian S, Bodey GP, et al. Randomized comparison between antibiotics alone and antibiotics plus granulocyte-macrophage colony-stimulating factor (Escherichia coli-derived) in cancer patients with fever and neutropenia. Am J Med 1996; 100:17-23.

146. Biesma B, deVries EG, Willemse PH. Efficacy and tolerability of recombinant human granulocyte-macrophage colony-stimulating factor in patients with chemotherapy-related leukopenia and fever. Eur J Cancer 1990; 26:932-6.

147. Ravaud A, Chevreau C, Cany L, et al. Granulocyte-macrophage colony-stimulating factor in patients with neutropenic chemotherapy regimens: results of a randomized phase III trial. J Clin Oncol 1998;16: 2930-6.

148. Maher DW, Lieschke GJ, Green M, et al. Filgrastim in patients with chemotherapy-induced febrile neutropenia - a double-blind, placebocontrolled trial. Ann Intern Med 1994; 121:492-501.

149. Vellenga E, Uyl-de Groot CA, de Wit R, et al. Randomized placebocontrolled trial of granulocyte-macrophage colony-stimulating factor in patients with chemotherapy related febrile neutropenia. J Clin Oncol 1996; 14:619-27.

150. Liang DC, Chen SH, Lean SF. Role of granulocyte colony-stimulating factor as adjunct therapy for septicemia in children with acute leukemia. Am J Hematol 1995; 48:76-81.

151. Mitchell PLR, Morland BJ, Dick G, et al. Clinical benefits and cost savings of interventional G-CSF therapy in patients with febrile neutropenia following chemotherapy [abstract]. Blood 1995; 86(Suppl 1): 500A.

152. Mayordomo JI, Rivera F, Diaz-Puente MT, et al. Improving treatment of chemotherapy-induced neutropenic fever by administration of colony-stimulating factors. J Natl Cancer Inst 1995; 87:803-8.

153. Mitchell PL, Morland B, Stevens MC, et al. Granulocyte colony-stimulating factor in established febrile neutropenia: a randomized study of pediatric patients. J Clin Oncol 1997; 15:1163-70.

154. Michon JM, Hartmann O, Boufett E, et al. An open-label, multicentre, randomised phase 2 study of recombinant human granulocyte colonystimulating factor (filgrastim) as an adjunct to combination chemotherapy in paediatric patients with metastatic neuroblastoma. Eur J Cancer 1998; 34:1063-9.

155. Yoshida M, Karasawa M, Naruse T, et al. Effect of granulocyte-colony stimulating factor on empiric therapy with flomoxef sodium and tobramycin in febrile neutropenic patients with hematological malignancies. Kan-etsu Hematological Disease and Infection Study Group. Int J Hematol 1999; 69:81-8.

156. Ozer H, Armitage JO, Bennett CL, et al. 2000 update of recommendations for the use of hematopoietic colony-stimulating factors: evidence-based clinical practice guidelines. J Clin Oncol 2000; 18 : $3558-85$. 
157. Hidalgo M, Hornedo J, Lumbreras C, et al. Lack of ability of ciprofloxacin-rifampin prophylaxis to decrease infection-related morbidity in neutropenic patients given cytotoxic therapy and peripheral blood stem cell transplants. Antimicrob Agents Chemother 1997; 41: 1175-7.

158. Engels EA, Lau J, Barza M. Efficacy of quinolone prophylaxis in neutropenic cancer patients: a meta-analysis. J Clin Oncol 1998; 16: 1179-87.

159. Munzo L, Martino R, Subira M, et al. Intensified prophylaxis of febrile neutropenia with ofloxacin plus rifampin during severe short-duration neutropenia in patient with lymphoma. Leuk Lymphoma 1999;34: 585-9.

160. Hughes WT, Rivera GK, Schell MJ, et al. Successful intermittent chemoprophylaxis for Pneumocystis carinii pneumonitis. N Engl J Med 1987; 316:1627-32.

161. Murphy M, Brown AE, Sepkowitz A, et al. Fluoroquinolone prophylaxis for the prevention of bacterial infections in patients with cancer-is it justified? Clin Infect Dis 1997; 25:346-7.

162. Kern W, Kurrle E. Ofloxacin versus trimethoprim-sulfamethoxazole for prevention of infection in patients with acute leukemia and granulocytopenia. Infection 1991; 19:73-80.

163. Bow EJ, Mandell LA, Louie TJ, et al. Quinolone-based chemoprophylaxis in neutropenic patients: effect of augmented gram-positive activity on infectious morbidity. Ann Intern Med 1996; 125:183-90.

164. Harvathova Z, Spanik S, Sufliarsky J, et al. Bacteremia due to methicillin-resistant staphylococci occurs more frequently in neutropenia patients who received antimicrobial prophylaxis and is associated with higher mortality in comparison of methicillin-sensitive bacteremia. Int J Antimicrob Agents 1998; 10:55-8.

165. International Antimicrobial Therapy Cooperative Group of the European Organization for Research and Treatment and Cancer. Reduction of fever and streptococcal bacteremia in granulocytopenic patients with cancer: a trial of oral penicillin $\mathrm{V}$ or placebo combined with pefloxacin. JAMA 1994;272:1183-9.

166. Cruciani M, Rampazzo R, Malena M, et al. Prophylaxis with fluoroquinolones in neutropenic patients: a meta-analysis. Clin Infect Dis 1996; 23:795-805.

167. Rotstein C, Mandell LA, Goldberg N. Fluoroquinolone prophylaxis for profoundly neutropenic cancer patients: a meta-analysis. Current Opinion in Oncology 1997; 4(Suppl 2):S2-7.

168. Carratala J, Fernandez-Sevilla A, Tubau F, et al. Emergence of quinolone-resistant Escherichia coli bacteremia in neutropenic patients with cancer who have received prophylactic norfloxacin. Clin Infect Dis 1995; 20:557-60.

169. Somolinos N, Arranz R, Del Rey MC, et al. Superinfections by Escherichia coli resistant to fluoroquinolones in immunocompromised patients [letter]. J Antimicrob Chemother 1992;30:730-1.

170. Kern WV, Andriof E, Oethinger M, et al. Emergence of fluoroquinolone-resistant Escherichia coli at a cancer center. Antimicrob Agents Chemother 1994; 38:681-7.

171. Cometta A, Calandra T, Bille J, et al. Escherichia coli resistant to fluoroquinolones in patients with cancer and neutropenia [letter]. N Engl J Med 1994;330:1240-1.

172. Goodman JL, Winston DJ, Greenfield A, et al. A controlled trial of fluconazole to prevent fungal infections in patients undergoing bone marrow transplantation. N Engl J Med 1992;326:845-51.

173. Ellis ME, Clink H, Ernst P, et al. Controlled study of fluconazole in the prevention of fungal infections in neutropenic patients with haematological malignancies and bone marrow transplant recipients. Eur J Clin Microbiol Infect Dis 1994; 13:3-11.
174. Marr KA, Seidel K, White TC, et al. Candidemia in allogenic blood and marrow transplant recipients: evolution of risk factors after the adoption of prophylactic fluconazole. J Infect Dis 2000; 181:309-16.

175. Wingard JR, Merz WG, Rinaldi MG, et al. Increase in Candida krusei infection among patients with bone marrow transplantation and neutropenia treated prophylactically with fluconazole. N Engl J Med 1991; 325:1274-7.

176. Rotstein C, Bow EG, Laverdiere M, et al. Randomized placebo-controlled trial of fluconazole prophylaxis for neutropenic patients: benefit based on purpose and intensity of cytotoxic therapy. Clin Infect Dis $1999 ; 28: 331-40$.

177. Centers for Disease Control and Prevention. Guidelines for preventing opportunistic infections among hematopoietic stem cell transplant recipients - recommendations of CDC, the Infectious Diseases Society of America, and the American Society of Blood and Transplantation. MMWR Morb Mortal Wkly Rep 2000; 49(RR-10):1-125.

178. Menichetti F, Flavero AD, Martion P, et al. Itraconazole oral solution as prophylaxis for fungal infections in neutropenic patients with hematologic malignancies: a randomized, placebo-controlled, doubleblind, multicenter trial. Clin Infect Dis 1999; 28:250-5.

179. Nucci M, Biasoli I, Akiti T, et al. A double-blind, randomized, placebocontrolled trial of itraconazole capsules as antifungal prophylaxis for neutropenic patients. Clin Infect Dis 2000; 30:300-5.

180. Bow EJ, Laverdiere M, Lussier N, Rotstein C. Antifungal prophylaxis in neutropenic patients: a meta-analysis of randomized-controlled trials [abstract 339A]. Blood 1999; 94(Suppl 1):339A.

181. Rolston KVI. Outpatient management of febrile, neutropenic patients. Infections in Medicine 1995; 12:12-5.

182. Talcott JA, Whalen A, Clark J. Home antibiotic therapy for low-risk cancer patients with fever and neutropenia: a pilot study of 30 patients based on a validated prediction rule. J Clin Oncol 1994; 12:107-14.

183. DiNubile MJ. Stopping antibiotic therapy in neutropenic patients. Ann Intern Med 1988; 108:289-92.

184. Lau RC, Doyle JJ, Freedman MH, et al. Early discharge of pediatric febrile neutropenic cancer patients by substitution of oral for intravenous antibiotics. Pediatr Hematol Oncol 1994; 11:417-21.

185. Mullen CA, Petropoulos D, Roberts WM, et al. Economic and resource utilization analysis of outpatient management of fever and neutropenia in low-risk pediatric patients with cancer. J Pediatr Hematol Oncol 1999;21:212-8.

186. deLalla F. Antibiotic treatment of febrile episodes in neutropenic cancer patients: clinical and economic considerations. Drugs 1997; 53: 789-804.

187. Klastersky J. Current attitudes for therapy for febrile neutropenia with consideration to cost-effectiveness. Curr Opin Oncol 1998; 10:284-90.

188. Gibson J, Johnson L, Snowdon L, et al. A randomized dosage study of ceftazidime with single daily tobramycin for the empirical management of febrile neutropenia in patients with hematological diseases. Int J Hematol 1994; 60:119-27.

189. Lyman GH, Kuderer NM, Bladucci L. Economic impact of granulopoiesis stimulating agents on the management of febrile neutropenia. Curr Opin Oncol 1998; 10:291-6.

190. Centers for Disease Control and Prevention. Assessing the effectiveness of disease and injury prevention programs: costs and consequences. MMWR Morb Mortal Wkly Rep 1995;44(RR-10):1-10.

191. Haddix A, Teutsch SM, Shaffer PS, Dunet D. Prevention effectiveness: a guide to decision analysis and economic evaluation. New York: Oxford University Press, 1996. 


\section{ERRATUM}

An error appeared in the Infectious Diseases Society of America guidelines published in the 15 March 2002 issue of the journal (Hughes WT, Armstrong D, Bodey GP, et al. 2002 Guidelines for the use of antimicrobial agents in neutropenic patients with cancer. Clin Infect Dis 2002;34:730-51). The authors listed in reference 41 should be "Hidalgo M, Hornedo J,
Lumbreras C, et al." (not "Garcia-Carbonero R, Cortes-Funes $\mathrm{H}$ "). The corrected version of reference 41 is as follows: "Hidalgo M, Hornedo J, Lumbreras C, et al. Outpatient therapy with oral ofloxacin for patients with low risk neutropenia and fever: a prospective, randomized clinical trial. Cancer 1999; 85: 213-9." The authors regret this error. 